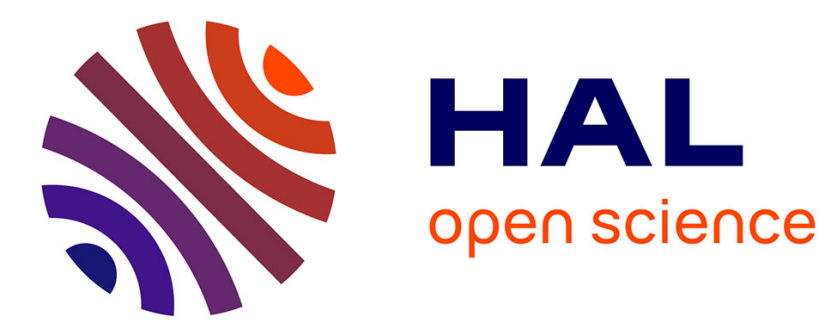

\title{
An overview of the modelling of fracture by gradient damage models
}

Jean-Jacques Marigo, Corrado Maurini, Kim Pham

\section{To cite this version:}

Jean-Jacques Marigo, Corrado Maurini, Kim Pham. An overview of the modelling of fracture by gradient damage models. Meccanica, 2016, 51 (12), pp.3107-3128. 10.1007/s11012-016-0538-4 . hal01374814

\section{HAL Id: hal-01374814 https://hal.science/hal-01374814}

Submitted on 1 Oct 2016

HAL is a multi-disciplinary open access archive for the deposit and dissemination of scientific research documents, whether they are published or not. The documents may come from teaching and research institutions in France or abroad, or from public or private research centers.
L'archive ouverte pluridisciplinaire HAL, est destinée au dépôt et à la diffusion de documents scientifiques de niveau recherche, publiés ou non, émanant des établissements d'enseignement et de recherche français ou étrangers, des laboratoires publics ou privés. 


\title{
An overview of the modelling of fracture by gradient damage models
}

\author{
Jean-Jacques Marigo ${ }^{\mathrm{a}, *}$, Corrado Maurini ${ }^{\mathrm{b}}$, Kim Pham $^{\mathrm{c}}$ \\ ${ }^{a}$ Laboratoire de Mécanique des Solides, Ecole Polytechnique, F-91128 Palaiseau, France \\ ${ }^{b}$ Institut Jean Le Rond d'Alembert, Sorbonne Universités, UPMC, Univ Paris 06, CNRS, UMR 7190, France. \\ ${ }^{c} I M S I A$, ENSTA ParisTech, CNRS, CEA, EDF, Université Paris-Saclay, 828 Boulevard des Maréchaux, 91762 \\ Palaiseau Cedex France
}

\begin{abstract}
The paper is devoted to gradient damage models which allow us to describe all the process of degradation of a body including the nucleation of cracks and their propagation. The construction of such model follows the variational approach to fracture and proceeds into two stages: (i) definition of the energy; (ii) formulation of the damage evolution problem. The total energy of the body is defined in terms of the state variables which are the displacement field and the damage field in the case of quasi-brittle materials. That energy contains in particular gradient damage terms in order to avoid too strong damage localizations. The formulation of the damage evolution problem is then based on the concepts of irreversibility, stability and energy balance. That allows us to construct homogeneous as well as localized damage solutions in a closed form and to illustrate the concepts of loss of stability, of scale effects, of damage localization, and of structural failure. Moreover, the variational formulation leads to a natural numerical method based on an alternate minimization algorithm. Several numerical examples illustrate the ability of this approach to account for all the process of fracture including a $3 \mathrm{D}$ thermal shock problem where the crack evolution is very complex.
\end{abstract}

Keywords: Fracture, Damage, phase field, variational methods, non-local models, stability principle, finite elements

\footnotetext{
${ }^{*}$ Corresponding author.

Email: marigo@lms.polytechnique.fr
} 


\section{Introduction}

Failure in brittle material often manifests in the form of cracks where the material "breaks" and the stiffness drops. In discrete approaches of failure, the cracks are modeled as surface of discontinuity of the displacement fields where possible jumps of the displacement field can occur. They are opposed to continuum approaches in which the crack is viewed as a damaged zone where a loss of stiffness continuously occurs along localized bands of non-zero thickness. The most widely accepted discrete crack model is the Griffith model, which assumes that the creation of the crack is done at expenses of a energy dissipation proportional to the crack surface, and that the crack propagation is not possible if this energy is larger than the elastic energy rewarded during a virtual crack propagation. In the end of the nineties, this energetic theory has been put in a precise mathematical setting by [19], allowing for the generalisation to the case of cracks of arbitrary shapes and with complex evolution in time. The key advantage of the Griffith model with respect to the damage model is its simplicity when the crack path is postulated in advance.

In damage models, the failure is described by means of an internal variable, the so-called damage variable, which allows to modulate the stiffness of the material. In local damage models, the material failure is controlled purely locally i.e. at a material point, by a critical stress which defines an elastic domain and a damage criterion as in plasticity. For standard damage models, it is shown that the quasi-static evolution of a damaging body can be recast into a variational formulation [31, 38] which consists in minimizing locally the total energy of the system under an irreversibility condition on the damage variable while enforcing an energy balance condition. For standard damage models, the total energy can be read as the sum of an elastic energy and a dissipated energy. For stress-hardening local damage models, the evolution problem of a damaging body is well-posed and the response is unique. On the other hand, for stress-softening local damage models which aim to capture complete failure, the evolution problem becomes ill-posed [8] and damage tends to concentrate into band of zero thickness, hence leading to the failure of the body without any dissipated energy. From the numerical point of view, such stress-softening models gives spurious mesh dependency of the results.

To overcome such drawbacks, a regularization of the stress-softening damage model is required. A possible choice is to regularize the model through the introduction of gradient terms of the damage variable $[35,36,15,30,9,39]$. In the variational approach of damage mechanics, such regularization can be achieved in an elegant way by adding to the local part of the total energy, a non-local counterpart depending on the gradient of the damage variable [39]. This results in the necessary emergence of a internal length in such regularized damage model. The consequences are of different kinds : the damage criterion becomes non-local and damage localizations has a finite thickness related to the internal length of the model, hence leading to size effects in the model response. Moreover, when the internal length is "small" compared to the characteristic size of the body, the gradient damage model behaves asymptotically as a Griffith fracture model with damage localizations viewed as sharp cracks $[14,46]$. However, compared 
to Griffith fracture model, such gradient damage models or alternatively called smeared crack models are much more practical when considering cracks of unknown and possibly complex shapes, because they do not require the explicit description of the crack geometry: the cracks are identified a posteriori as the regions where the elastic stiffness vanishes over localized bands. Another fundamental advantage of gradient damage models is related to the existence an intrinsic elastic limit stress compared to Griffith model and therefore the ability to retrieve crack nucleation, i.e. the creation of a crack from an intact material with smooth boundaries.

The fundamental link between gradient damage models and Griffith model of fracture mechanics relies strongly on the variational structure of both models, hence justifying the necessity to work with standard damage models. Indeed, gradient damage models appear as an elliptic approximation of the variational fracture mechanics problem. The variational approach of brittle fracture recast the evolution problem for the cracked state of a body as a minimality principle for an energy functional sum of the elastic energy and the energy dissipated to create the crack [19]. On the basis of the results of the mathematical theory of image segmentation and free-discontinuity problems [34, 3], [12] approximate the minima of this energy functional through the minimization of a regularized elliptic functional that may be mechanically interpreted as the energy of a gradient damage model with an internal length. Mathematical results based on Gamma-Convergence theory show that when the internal length of a large class of gradient damage models tends to zero, the global minima of the damage energy functional tend towards the global minima of the energy functional of Griffith brittle fracture [14]. The same is true for the corresponding quasi-static evolutions ruled by a global minimality principle [22]. Similar variational approximations of brittle fracture have emerged in the community of physicists adopting Ginzburg-Landau theories to study phase transitions [25, 23], producing relevant results [43]. Nowadays the so-called phase-field models of fracture are extensively adopted in computational mechanics to study fracture phenomena [27, 20, 44, 26, 33, 10, 28].

This text focusses on the fundamental variational principles which govern the construction and the main properties of gradient damage models. It constitutes essentially a summary of the works devoted to the variational approach to fracture, starting from [19] and followed by several other papers like [12] where the first numerical tests using Ambrosio-Tortorelli's model are presented, $[38,39]$ where a general method for the construction of gradient damage models is proposed, [42] where a method for the experimental identification of a gradient damage model is proposed, [9] or [40, 41] where some general qualitative properties of such models are discussed, [4] where the delicate issue of the asymmetric behavior between tension and compression is addressed, [46] where a fundamental link with Griffith's fracture theory is proved, [47] and [13] where a complete theoretical and numerical analysis of the thermal shock problem is made and compared to experimental results.

The paper is organized as follows. Section 2 is devoted to the formulation of gradient damage models in a general context. In Section 3, we consider the evolution problem for the 1D traction of a bar. We first construct the homogeneous solution before to study its stability. Then we 
construct solutions with damage localization. Finally we compare several models on the basis of two fundamental global properties: the force-displacement curve and the stability diagram for homogenous solutions. This analysis provides useful criteria for the selection of the constitutive laws and the identification of the internal length. Section 4 describes the numerical strategy that may be adopted to solve the damage evolution problem in a general setting. In particular, the numerical results obtained for the $2 \mathrm{D}$ version of the traction test are compared to the analytical ones derived in the 1D setting. We finally consider the thermal shock problem to illustrate all the power of the variational approach to fracture by gradient damage models. In particular, it is shown that one can account for all stages of the fracture evolution, from the nucleation of an array of periodic cracks to the phenomenon of period doubling, in perfect agreement both qualitatively and quantitatively with experimental results.

The summation convention on repeated indices is implicitly adopted in the sequel. The vectors and second order tensors are indicated by boldface letters, like $\mathbf{u}$ and $\boldsymbol{\sigma}$ for the displacement field and the stress field. Their components are denoted by italic letters, like $u_{i}$ and $\sigma_{i j}$. The third or fourth order tensors as well as their components are indicated by a sans serif letter, like $\mathrm{A}$ or $\mathrm{A}_{i j k l}$ for the stiffness tensor. Such tensors are considered as linear maps applying on vectors or second order tensors and the application is denoted without dots, like $A \varepsilon$ whose $i j$-component is $\mathrm{A}_{i j k l} \varepsilon_{k l}$. The inner product between two vectors or two tensors of the same order is indicated by a dot, like $\mathbf{a} \cdot \mathbf{b}$ which stands for $a_{i} b_{i}$ or $\boldsymbol{\sigma} \cdot \boldsymbol{\varepsilon}$ for $\sigma_{i j} \varepsilon_{i j}$. We use the notation $\mathrm{A}>0$ to denote positive definite tensor.

\section{Variational formulation of isotropic damage models}

\subsection{Setting of the gradient damage model}

We simply recall here the main steps of the construction of a gradient damage model by a variational approach, the reader interested by more details should refer to [38] and [39]. Let us consider a homogeneous $n$-dimensional body whose reference configuration is the open connected bounded set $\Omega \subset \mathbb{R}^{n}$ of characteristic size $L$. This body is made of a strongly brittle damaging material whose behavior is defined as follows:

1. The damage parameter is a scalar which can only grow from 0 to $1, \alpha=0$ denoting the undamaged state and $\alpha=1$ the completely damaged state.

2. The state of the volume element is characterized by the triplet $(\varepsilon, \alpha, \mathbf{g})$ where $\varepsilon, \alpha$ and $\mathbf{g}$ denote respectively the strain tensor, the damage parameter and the gradient of damage vector $(\mathbf{g}=\nabla \alpha)$.

3. The bulk energy density of the material is the state function $W:(\varepsilon, \alpha, \mathbf{g}) \mapsto W(\varepsilon, \alpha, \mathbf{g})$. Therefore, the material behavior is non local in the sense that it depends on the gradient of damage. To simplify the presentation, we will only consider behaviors such that the 
bulk energy density is the sum of three terms: the stored elastic energy $\psi(\varepsilon, \alpha)$, the local part of the dissipated energy by damage $w(\alpha)$ and its non local part $\frac{1}{2} w_{1} \ell^{2} \mathbf{g} \cdot \mathbf{g}$,

$$
W(\varepsilon, \alpha, \mathbf{g})=\psi(\varepsilon, \alpha)+w(\alpha)+\frac{1}{2} w_{1} \ell^{2} \mathbf{g} \cdot \mathbf{g},
$$

each of these terms enjoying the following properties:

(a) The elastic energy reads as

$$
\psi(\varepsilon, \alpha)=\frac{1}{2} \mathrm{~A}(\alpha)\left(\varepsilon-\varepsilon^{0}\right) \cdot\left(\varepsilon-\varepsilon^{0}\right),
$$

where $\varepsilon^{0}$ is a given pre-strain ${ }^{1}$ and $\mathrm{A}(\alpha)$ is the stiffness tensor of the material in its damaged state $\alpha$. The stiffness function $\alpha \mapsto \mathrm{A}(\alpha)$ decreases from $\mathrm{A}_{0}$ to 0 when $\alpha$ grows from 0 to 1 .

(b) The local dissipated energy density is a positive increasing function of $\alpha$, increasing from 0 when $\alpha=0$ to a finite positive value $w_{1}$ when $\alpha=1$. Therefore $w_{1}$ represents the energy dissipated during a complete, homogeneous damage process of a volume element: $w_{1}=w(1)$.

(c) The non local dissipated energy density is assumed to be a quadratic function of the gradient of damage. Since the damage parameter is dimensionless and by virtue of the above definition of $w_{1}, \ell$ has the dimension of a length. Accordingly, $\ell$ can be considered as an internal length characteristic of the material while having always in mind that the definition of $\ell$ depends on the normalizations associated with the choices of the critical value 1 for $\alpha$ and $w(1)$ for the multiplicative factor.

4. The dual quantities associated with the state variables are respectively the stress tensor $\boldsymbol{\sigma}$, the energy release rate density $\mathrm{Y}$ and the damage flux vector $\mathbf{q}$ :

$$
\boldsymbol{\sigma}=\frac{\partial W}{\partial \boldsymbol{\varepsilon}}(\varepsilon, \alpha, \mathbf{g}), \quad \mathbf{Y}=-\frac{\partial W}{\partial \alpha}(\varepsilon, \alpha, \mathbf{g}), \quad \mathbf{q}=\frac{\partial W}{\partial \mathbf{g}}(\varepsilon, \alpha, \mathbf{g})
$$

Accordingly, these dual quantities are given by the following functions of state:

$$
\boldsymbol{\sigma}=\mathrm{A}(\alpha)\left(\varepsilon-\varepsilon^{0}\right), \quad \mathrm{Y}=-\frac{1}{2} \mathrm{~A}^{\prime}(\alpha)\left(\varepsilon-\varepsilon^{0}\right) \cdot\left(\varepsilon-\varepsilon^{0}\right)-w^{\prime}(\alpha), \quad \mathbf{q}=w_{1} \ell^{2} \mathbf{g},
$$

where the prime denotes the derivative with respect to $\alpha$.

The underlying local behavior is characterized by the function $W_{0}$ defined by $W_{0}(\varepsilon, \alpha)=$ $W(\varepsilon, \alpha, \mathbf{0})$. We assume that it corresponds to a strongly brittle material, see [40, Hypothesis 1$]$. That means specifically that the material has a softening behavior but also that the energy dissipated during a process where the damage parameter grows from 0 to 1 is finite. The latter property is ensured by the fact that $w(1)<+\infty$. The former one requires that the elastic

\footnotetext{
${ }^{1}$ In the thermal shock problem treated in the last section, the pre-strain is the thermal strain induced by the given temperature field which depends on time. Therefore, we will assume that $\varepsilon^{0}$ is a given time-dependent field.
} 
domain in the strain space $\mathcal{R}(\alpha)$ is an increasing function of $\alpha$ (strain hardening) while the elastic domain in the stress space $\mathcal{R}^{*}(\alpha)$ is a decreasing function of $\alpha$ (stress softening). Those elastic domains are defined by

$$
\mathcal{R}(\alpha)=\left\{\varepsilon \in \mathbb{M}_{s}: \frac{\partial W_{0}}{\partial \alpha}(\varepsilon, \alpha) \geq 0\right\}, \quad \mathcal{R}^{*}(\alpha)=\left\{\boldsymbol{\sigma} \in \mathbb{M}_{s}: \frac{\partial W_{0}^{*}}{\partial \alpha}(\boldsymbol{\sigma}, \alpha) \leq 0\right\}
$$

where $W_{0}^{*}(\boldsymbol{\sigma}, \alpha)=\sup _{\boldsymbol{\varepsilon} \in \mathbb{M}_{s}}\left\{\boldsymbol{\sigma} \cdot \boldsymbol{\varepsilon}-W_{0}(\boldsymbol{\varepsilon}, \alpha)\right\}$ and $\mathbb{M}_{s}$ denotes the space of symmetric tensors.

In the present context, one gets

$$
W_{0}(\varepsilon, \alpha)=\frac{1}{2} \mathrm{~A}(\alpha)\left(\varepsilon-\varepsilon^{0}\right) \cdot\left(\varepsilon-\varepsilon^{0}\right)+w(\alpha)
$$

and hence

$$
W_{0}^{*}(\boldsymbol{\sigma}, \alpha)=\boldsymbol{\sigma} \cdot \varepsilon^{0}+\frac{1}{2} \mathrm{~S}(\alpha) \boldsymbol{\sigma} \cdot \boldsymbol{\sigma}-w(\alpha),
$$

where the compliance tensor $\mathrm{S}(\alpha)=\mathrm{A}(\alpha)^{-1}$ increases from $\mathrm{S}_{0}$ to infinity when $\alpha$ grows from 0 to 1 . Accordingly, the elastic domains $\mathcal{R}(\alpha)$ and $\mathcal{R}^{*}(\alpha)$ read

$$
\begin{aligned}
\mathcal{R}(\alpha) & =\left\{\boldsymbol{\varepsilon} \in \mathbb{M}_{s}:-\frac{1}{2} \mathrm{~A}^{\prime}(\alpha)\left(\varepsilon-\varepsilon^{0}\right) \cdot\left(\varepsilon-\varepsilon^{0}\right) \leq w^{\prime}(\alpha)\right\} \\
\mathcal{R}^{*}(\alpha) & =\left\{\boldsymbol{\sigma} \in \mathbb{M}_{s}: \frac{1}{2} \mathrm{~S}^{\prime}(\alpha) \boldsymbol{\sigma} \cdot \boldsymbol{\sigma} \leq w^{\prime}(\alpha)\right\} .
\end{aligned}
$$

Therefore, the material is said to be

- strain hardening when $\alpha \mapsto\left(-\mathrm{A}^{\prime}(\alpha) / w^{\prime}(\alpha)\right)$ is decreasing with respect to $\alpha$, i.e.:

$$
w^{\prime}(\alpha) \mathrm{A}^{\prime \prime}(\alpha)-w^{\prime \prime}(\alpha) \mathrm{A}^{\prime}(\alpha)>0 \text {. }
$$

This means that the domain of admissible strains in the elastic regime is increasing for increasing damage.

- stress hardening (resp. softening) when $\alpha \mapsto\left(\mathrm{S}^{\prime}(\alpha) / w^{\prime}(\alpha)\right)$ is decreasing (resp. increasing) with respect to $\alpha$, i.e.:

$$
w^{\prime}(\alpha) S^{\prime \prime}(\alpha)-w^{\prime \prime}(\alpha) S^{\prime}(\alpha)<(\text { resp. }>) 0,
$$

then the domain of admissible stress in the elastic regime is increasing (resp. decreasing) for increasing damage.

The critical stress $\sigma_{c}$ in a uniaxial tensile test such that $\boldsymbol{\sigma}=\sigma_{c} \mathbf{e}_{1} \otimes \mathbf{e}_{1}$ is then given by

$$
\sigma_{c}=\sqrt{\frac{2 w^{\prime}(0)}{\mathrm{S}_{1111}^{\prime}(0)}} .
$$

Remark 1. The present model is a brittle damage model without any plasticity-like effects. Including plastic behaviors in our formulation is not a major difficulty. It would require to change the form of the strain energy by introducing plastic strains and by adding a plastic dissipated energy (possibly damage dependent), see [1, 2]. 


\subsection{The evolution problem}

The body is submitted to a time dependent loading which consists of a density of volume forces $\mathbf{f}_{t}$, a density of surface forces $\mathbf{F}_{t}$ prescribed on the part $\partial_{N} \Omega$ of the boundary and prescribed displacements $\mathbf{U}_{t}$ on the complementary part $\partial_{D} \Omega$ of the boundary, $t$ denoting the time parameter. The potential of the given external forces at time $t$ can read as the following linear form $\mathcal{W}_{t}^{e}$ defined on the set $\mathcal{C}_{t}$ of kinematically admissible displacement fields

$$
\mathcal{W}_{t}^{e}(\mathbf{v}):=\int_{\Omega} \mathbf{f}_{t} \cdot \mathbf{v} \mathrm{d} x+\int_{\partial_{N} \Omega} \mathbf{F}_{t} \cdot \mathbf{v} \mathrm{d} s
$$

with

$$
\mathcal{C}_{t}:=\left\{\mathbf{v}: \mathbf{v}=\mathbf{U}_{t} \text { on } \partial_{D} \Omega\right\}
$$

The law of evolution of the damage in the body is written in a variational form and based on the definition of the total energy of the body associated with admissible states. Specifically, if $(\mathbf{v}, \beta)$ denotes a pair of admissible displacement and damage fields at time $t$, i.e. if $\mathbf{v} \in \mathcal{C}_{t}$ and $\beta \in \mathcal{D}$ with

$$
\mathcal{D}:=\{\beta: 0 \leq \beta \leq 1 \text { in } \Omega\},
$$

then the total energy of the body at time $t$ in this state is given by

$$
\mathcal{P}_{t}(\mathbf{v}, \beta):=\int_{\Omega} W(\varepsilon(\mathbf{v}), \beta, \nabla \beta) \mathrm{d} x-\mathcal{W}_{t}^{e}(\mathbf{v})
$$

where $\boldsymbol{\varepsilon}(\mathbf{v})$ denotes the symmetrized gradient of $\mathbf{v}$.

The damage evolution law. Following the variational approach presented in [38, 39], the evolution of the damage in the body is governed by the three principles of irreversibility, stability and energy balance. Specifically these conditions read as follows:

1. Irreversibility: $t \mapsto \alpha_{t}$ must be non decreasing and, at each time $t \geq 0, \alpha_{t} \in \mathcal{D}$.

2. Stability: At each time $t>0$, the real state $\left(\mathbf{u}_{t}, \alpha_{t}\right)$ must be stable in the sense that for all $\mathbf{v} \in \mathcal{C}_{t}$ and all $\beta \in \mathcal{D}$ such that $\beta \geq \alpha_{t}$, there exists $\bar{h}>0$ such that for all $h \in[0, \bar{h}]$

$$
\mathcal{P}_{t}\left(\mathbf{u}_{t}+h\left(\mathbf{v}-\mathbf{u}_{t}\right), \alpha_{t}+h\left(\beta-\alpha_{t}\right) \geq \mathcal{P}_{t}\left(\mathbf{u}_{t}, \alpha_{t}\right) .\right.
$$

3. Energy balance: At each time $t>0$, assuming that the pre-strain does not evolve, the following energy balance must hold:

$$
\mathcal{P}_{t}\left(\mathbf{u}_{t}, \alpha_{t}\right)=\mathcal{P}_{0}\left(\mathbf{u}_{0}, \alpha_{0}\right)+\int_{0}^{t}\left(\int_{\Omega} \boldsymbol{\sigma}_{s} \cdot\left(\varepsilon\left(\dot{\mathbf{U}}_{s}\right)-\dot{\varepsilon}_{s}^{0}\right) \mathrm{d} x-\mathcal{W}_{s}^{e}\left(\dot{\mathbf{U}}_{s}\right)-\dot{\mathcal{W}}_{s}^{e}\left(\mathbf{u}_{s}\right)\right) \mathrm{d} s
$$

In (13), $\alpha_{0}$ denotes the given damage state at the beginning of the loading process whereas $\mathbf{u}_{0}$ is the associated displacement field obtained by solving the elastostatic problem at time 0 : $\mathbf{u}_{0}=\operatorname{argmin}_{\mathbf{v} \in \mathcal{C}_{0}} \mathcal{P}_{0}\left(\mathbf{v}, \alpha_{0}\right) ; \boldsymbol{\sigma}_{s}$ denotes the real stress field at time $s$ and is given by $(3), \dot{\mathbf{U}}_{s}$ is the rate of a given (but arbitrarily chosen) admissible displacement field at time $s, \dot{\varepsilon}_{s}^{0}$ is the given rate of the prescribed pre-strain at time $s$ and $\dot{\mathcal{W}}_{s}^{e}$ denotes the linear form associated with the rate of the prescribed volume or surface forces at time $s$. 


\subsection{Kuhn-Tucker necessary conditions}

Throughout the paper we will assume that the fields are sufficiently smooth so that all the calculations make sense. A precise statement of the functional spaces which are needed to justify all steps is a very difficult task and remains far from the scope of the present paper. A natural requirement is that the total energy remains finite at each time. Accordingly, the damage field cannot be discontinuous across any surface. The question is more delicate for the displacement field because of the loss of stiffness at the points where $\alpha$ is equal to 1 . To simplify the presentation, we only consider in the present subsection the first stage of the damage evolution, before the nucleation of a crack. In other words, we assume that $\alpha_{t}<1$ everywhere in the body. The reader interested by the case where a crack has nucleated and propagates should refer to [46] where a complete analysis is made.

One immediately deduces that the stability condition (12) is satisfied only if, at each time, the body is at equilibrium and the damage criterion is satisfied. Specifically, these two conditions respectively read in a variational form as

$$
\begin{gathered}
\int_{\Omega} \boldsymbol{\sigma}_{t} \cdot \boldsymbol{\varepsilon}\left(\mathbf{v}-\mathbf{u}_{t}\right) \mathrm{d} x=\mathcal{W}_{t}^{e}\left(\mathbf{v}-\mathbf{u}_{t}\right), \quad \forall \mathbf{v} \in \mathcal{C}_{t}, \\
\int_{\Omega}\left(-\mathrm{Y}_{t} \cdot\left(\beta-\alpha_{t}\right)+\mathbf{q}_{t} \cdot \nabla\left(\beta-\alpha_{t}\right)\right) \mathrm{d} x \geq 0, \quad \forall \beta \in \mathcal{D}: \beta \geq \alpha_{t},
\end{gathered}
$$

where $\boldsymbol{\sigma}_{t}, \mathrm{Y}_{t}$ and $\mathbf{q}_{t}$ denote respectively the stress tensor, the energy release rate density and damage flux vector which are given in terms of the current state by the constitutive relations (3). These two conditions can be seen as the first order stability conditions. They are necessary but not always sufficient in order for (12) to hold. We will introduce second order stability conditions in the next section to study the stability of the homogeneous response of a bar under traction.

By standard arguments of the Calculus of Variations, one obtains that (14) is equivalent for the stress $\boldsymbol{\sigma}_{t}=\mathrm{A}(\alpha) \boldsymbol{\varepsilon}\left(\mathbf{u}_{t}\right)$ to satisfy the local equilibrium equation and the natural boundary conditions on the part of the boundary where they are prescribed:

$$
\begin{aligned}
& \operatorname{div} \boldsymbol{\sigma}_{t}+\mathbf{f}_{t}=0 \quad \text { in } \quad \Omega, \\
& \boldsymbol{\sigma}_{t} \cdot \mathbf{n}=\mathbf{F}_{t} \quad \text { on } \quad \partial_{F} \Omega .
\end{aligned}
$$

By the same procedure, one obtains that (15) is equivalent to the inequalities $\mathrm{Y}_{t}+\operatorname{div} \mathbf{q}_{t} \leq 0$ in $\Omega$ and $\mathbf{q}_{t} \cdot \mathbf{n} \geq 0$ on $\partial \Omega$. Using the definitions of the different terms, that leads to the following inequalities:

$$
\begin{gathered}
0 \leq-\frac{1}{2} \mathrm{~S}^{\prime}\left(\alpha_{t}\right) \boldsymbol{\sigma}_{t} \cdot \boldsymbol{\sigma}_{t}+w^{\prime}\left(\alpha_{t}\right)-w_{1} \ell^{2} \Delta \alpha_{t} \quad \text { in } \quad \Omega \\
0 \leq \frac{\partial \alpha_{t}}{\partial n} \quad \text { on } \quad \partial \Omega
\end{gathered}
$$


The inequality (18) is nothing but the non local damage criterion which involves the laplacian of the damage field because of the presence of the gradient damage term in the energy. However, one can see that it gives the usual local criterion in the part of the body which is still undamaged at time $t$. Indeed, in the interior of the domain where $\alpha_{t}=0,(18)$ reads as $\frac{1}{2} S^{\prime}(0) \sigma_{t} \cdot \sigma_{t} \leq w^{\prime}(0)$, which requires that the stress field is bounded.

Let us use now the energy balance (13). Assuming that the evolution is smooth in time and space, one can take the derivative of (13) with respect to $t$ which leads to

$$
\begin{aligned}
0 & =\frac{d}{d t} \mathcal{P}_{t}\left(\mathbf{u}_{t}, \alpha_{t}\right)-\int_{\Omega} \boldsymbol{\sigma}_{t} \cdot\left(\varepsilon\left(\dot{\mathbf{U}}_{t}\right)-\dot{\varepsilon}_{t}^{0}\right) \mathrm{d} x+\mathcal{W}_{t}^{e}\left(\dot{\mathbf{U}}_{t}\right)+\dot{\mathcal{W}}_{t}^{e}\left(\mathbf{u}_{t}\right) \\
& =\frac{d}{d t}\left(\int_{\Omega} W_{t} \mathrm{~d} x\right)-\int_{\Omega} \boldsymbol{\sigma}_{t} \cdot\left(\varepsilon\left(\dot{\mathbf{U}}_{t}\right)-\dot{\varepsilon}_{t}^{0}\right) \mathrm{d} x-\mathcal{W}_{t}^{e}\left(\dot{\mathbf{u}}_{t}-\dot{\mathbf{U}}_{t}\right),
\end{aligned}
$$

where $W_{t}=W\left(\varepsilon\left(\mathbf{u}_{t}\right), \alpha_{t}, \nabla \alpha_{t}\right)$. Let us evaluate the derivative with respect to $t$ of the bulk energy. By virtue of the regularity assumption, one gets

$$
\frac{d}{d t}\left(\int_{\Omega} W_{t} \mathrm{~d} x\right)=\int_{\Omega}\left(\boldsymbol{\sigma}_{t} \cdot\left(\varepsilon\left(\dot{\mathbf{u}}_{t}\right)-\dot{\varepsilon}_{t}^{0}\right)-\mathrm{Y}_{t} \cdot \dot{\alpha}_{t}+\mathbf{q}_{t} \cdot \nabla \dot{\alpha}_{t}\right) \mathrm{d} x .
$$

Inserting (21) into (20) leads to

$$
0=\int_{\Omega}\left(\boldsymbol{\sigma}_{t} \cdot \varepsilon\left(\dot{\mathbf{u}}_{t}-\dot{\mathbf{U}}_{t}\right)-\mathbf{f}_{t} \cdot\left(\dot{\mathbf{u}}_{t}-\dot{\mathbf{U}}_{t}\right)-\mathbf{Y}_{t} \cdot \dot{\alpha}_{t}+\mathbf{q}_{t} \cdot \nabla \dot{\alpha}_{t}\right) \mathrm{d} x-\int_{\partial_{N} \Omega} \mathbf{F}_{t} \cdot\left(\dot{\mathbf{u}}_{t}-\dot{\mathbf{U}}_{t}\right) \mathrm{d} s .
$$

Integrating by parts the gradient terms in the integrals over $\Omega$ in (22) and using the equilibrium equations (16)-(17) allow to simplify the energy balance which can read now

$$
0=-\int_{\Omega}\left(\mathrm{Y}_{t}+\operatorname{div} \mathbf{q}_{t}\right) \cdot \dot{\alpha}_{t} \mathrm{~d} x+\int_{\partial \Omega} \mathbf{q}_{t} \cdot \mathbf{n} \dot{\alpha}_{t} \mathrm{~d} s
$$

Since the irreversibility condition requires that $\dot{\alpha}_{t} \geq 0$ everywhere, since the damage criterion (18) gives $Y_{t}+\operatorname{div} \mathbf{q}_{t} \leq 0$ everywhere and since (19) gives $\mathbf{q}_{t} \cdot \mathbf{n} \geq 0$ on the boundary, the right hand side of (23) is the sum of non negative terms. Therefore, each term must vanish and we obtain the following consistency condition which can be seen as the local form of the energy balance:

$$
\begin{gathered}
\dot{\alpha}_{t}\left(-\frac{1}{2} \mathrm{~S}^{\prime}\left(\alpha_{t}\right) \boldsymbol{\sigma}_{t} \cdot \boldsymbol{\sigma}_{t}+w^{\prime}\left(\alpha_{t}\right)-w_{1} \ell^{2} \Delta \alpha_{t}\right)=0 \text { in } \Omega \\
\dot{\alpha}_{t} \frac{\partial \alpha_{t}}{\partial n}=0 \quad \text { on } \partial \Omega
\end{gathered}
$$

Let us remark that (24) states that, at each point, the damage level can increase only if the damage yield criterion is attained, i.e. only if (18) is an equality. We have thus proved that the variational formulation of the evolution problem based on the stability criterion, the energy balance and the irreversibility condition implies the equilibrium and the usual Kuhn-Tucker conditions. Note however that the equilibrium equations (16)-(17), the damage criterion (18)(19) and the local energy balances (24)-(25) are only necessary conditions. An evolution which satisfies those conditions has also to satisfy second order stability conditions to be really a solution. We will show that in the next sections. 


\section{Application to the $1 \mathrm{D}$ tension test}

\subsection{The $1 D$ problem}

This section is devoted to the evolution problem for the traction of a one-dimensional bar of length $L$, made of a homogeneous material with stress softening and without pre-strain $\left(\varepsilon^{0}=0\right)$. The end $x=0$ is fixed while the end $x=L$ has a displacement $U_{t}=t L$ imposed by a hard device (see Figure 1). This fundamental problem will allow us to illustrate some general properties of the variational approach to fracture by gradient damage models like

1. The role of a critical stress on the onset of damage;

2. Size effects due to the presence of a material characteristic length, the evolution of the damage being strongly dependent on the length of the bar;

3. The process of nucleation of a crack in long bars due to the stress softening which induces a damage localization;

4. The role of the gradient damage terms which prohibit any spatial jump of the damage field and lead to a finite value of the energy dissipated in the nucleation of a crack.

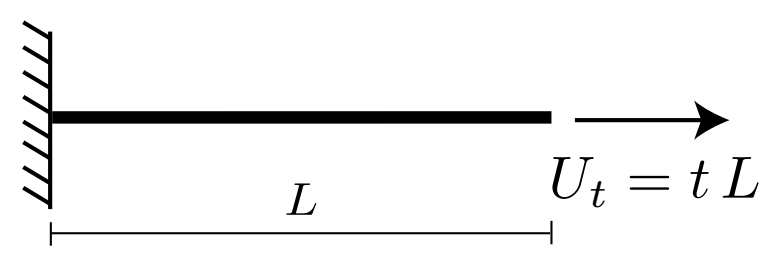

Figure 1: 1D traction problem with imposed end-displacement.

An admissible displacement field $u$ must satisfy the boundary conditions

$$
u(x=0)=0, \quad u(x=L)=U_{t}, \quad U_{0}=0 .
$$

Since no force is prescribed, the total energy (11), which does not depend explicitly on time, reads now as

$$
\mathcal{P}(u, \alpha)=\int_{0}^{L}\left(\frac{1}{2} E(\alpha) u^{\prime 2} d x+w(\alpha)+\frac{1}{2} w_{1} \ell^{2} \alpha^{\prime 2}\right) d x,
$$

where $E(\alpha)$ is the one-dimensional axial stiffness and $(\cdot)^{\prime}=\partial(\cdot) / \partial x$. Consequently, the equilibrium equation (16) becomes:

$$
\sigma_{t}^{\prime}(x)=0, \quad \sigma_{t}(x)=E\left(\alpha_{t}(x)\right) u_{t}^{\prime}(x), \quad \forall x \in(0, L) .
$$


Thus, the stress $\sigma_{t}$ along the bar is necessarily constant. Using the boundary conditions (26), we get

$$
\sigma_{t}=\frac{U_{t}}{\int_{0}^{L} S\left(\alpha_{t}(x)\right) d x},
$$

with $S(\alpha)=1 / E(\alpha)$. Moreover the set of necessary Kuhn-Tucker conditions read as

Irreversibility :

Damage criterion:

Energy balance :

Boundary conditions :

$$
\begin{aligned}
& \dot{\alpha}_{t} \geq 0, \quad \alpha_{0}=0, \\
& -w_{1} \ell^{2} \alpha_{t}^{\prime \prime}+\frac{1}{2} E^{\prime}\left(\alpha_{t}\right) u_{t}^{\prime 2}+w^{\prime}\left(\alpha_{t}\right) \geq 0, \\
& \dot{\alpha}_{t}\left(-w_{1} \ell^{2} \alpha_{t}^{\prime \prime}+\frac{1}{2} E^{\prime}\left(\alpha_{t}\right) u_{t}^{\prime 2}+w^{\prime}\left(\alpha_{t}\right)\right)=0, \\
& \alpha_{t}^{\prime}(0) \leq 0, \quad \alpha_{t}^{\prime}(L) \geq 0 .
\end{aligned}
$$

As we will see below, depending on the ratio $L / \ell$ between the length of the bar and the internal length, this set of relations admit either a unique homogeneous solution or an infinite number of solutions. In any case, one has to use the full stability criterion (12) to keep only the physically admissible solutions.

\subsection{The homogeneous evolution and its stability}

We consider only the case of strain hardening materials and hence assume that $\alpha \mapsto w^{\prime}(\alpha) / E^{\prime}(\alpha)$ is decreasing. We focus here on the study of the homogeneous evolution $\left(t x, \alpha_{t}\right)$ in which the damage and the strain fields are uniform all along the bar. By virtue of the boundary conditions, the solution of the elastic problem (28) takes the form:

$$
u_{t}^{\prime}(x)=t, \quad u_{t}(x)=t x, \quad \sigma_{t}=t E\left(\alpha_{t}\right) .
$$

Since the damage is also uniform in the bar, the damage criterion (30b) and the local energy balance (30c) become

$$
\frac{t^{2}}{2} E^{\prime}\left(\alpha_{t}\right)+w^{\prime}\left(\alpha_{t}\right) \geq 0, \quad \dot{\alpha}_{t}\left(\frac{t^{2}}{2} E^{\prime}\left(\alpha_{t}\right)+w^{\prime}\left(\alpha_{t}\right)\right)=0 .
$$

Since the bar is undamaged at the beginning of the loading $\left(\alpha_{0}=0\right)$, the solution of the evolution problem is characterized by an elastic phase and a damaging phase, as detailed below.

\subsubsection{Elastic phase}

For $t$ increasing from 0 , the damage criterion (30b) is a strict inequality if the end displacement $U_{t}$ is smaller than the elastic limit

$$
U_{e}=L \sqrt{-\frac{2 w^{\prime}(0)}{E^{\prime}(0)}}, \quad \text { with } \quad \sigma_{e}=L \sqrt{\frac{2 w^{\prime}(0)}{S^{\prime}(0)}} .
$$


The stress value $\sigma_{e}$ can be interpreted as the elastic limit stress. If $w^{\prime}(0)>0$, then $U_{e}>0$ and the material has a genuine elastic phase: as long as $U_{t}$ lies in the interval $\left[0, U_{e}\right]$, the material remains sound $\left(\alpha_{t}=0\right)$ with a stiffness $E_{0}=E(0)$ and the stress is given by $\sigma_{t}=E_{0} U_{t} / L$. On the other hand, if $w^{\prime}(0)=0$, then the damage criterion becomes an equality at the onset of the loading and we cannot observe an elastic phase.

\subsubsection{Damaging phase}

For $U_{t} \geq U_{e}$ the damage criterion (32) becomes an equality and the damage can grow. Hence, (32) gives the following implicit relation between the prescribed displacement $U_{t}$ and the associated homogeneous damage $\alpha_{t}$ :

$$
\frac{U_{t}}{L}=\sqrt{-\frac{2 w^{\prime}\left(\alpha_{t}\right)}{E^{\prime}\left(\alpha_{t}\right)}} .
$$

Owing to the strain hardening assumption, $\left(-w^{\prime} / E^{\prime}\right)$ is a monotonic increasing function. Thus, there is a unique solution $\alpha_{t}$ for a given $U_{t}$. In other words, the strain hardening hypothesis rules out snap-back phenomena during the evolution in time of homogeneous solutions. The corresponding stress is

$$
\sigma_{t}=E\left(\alpha_{t}\right) t=\sqrt{\frac{2 w^{\prime}\left(\alpha_{t}\right)}{S^{\prime}\left(\alpha_{t}\right)}} .
$$

Equation (35) clearly shows that the stress decreases (resp. grows) with the displacement if the material is stress softening (resp. stress hardening) at the damage level $\alpha_{t}$.

Remark 2 (Peak Stress). Since, by hypothesis, $w^{\prime}$ and $S^{\prime}$ are positive continuous functions on $\alpha \in[0,1)$, we can define the peak stress in the homogeneous response as

$$
\sigma_{M}=\sup _{\alpha \in[0,1)} \sqrt{\frac{2 w^{\prime}(\alpha)}{S^{\prime}(\alpha)}} .
$$

When $\sigma_{M}<+\infty$, then $\sigma_{M}$ is the maximal stress that the material can sustain. In particular, in the case of stress softening laws, the peak stress (36) is attained for $\alpha=0$ and is equal to the elastic limit stress $\sigma_{e}$.

\subsubsection{Stability of the homogeneous response and size effects}

Although the homogeneous strain-damage evolution $\left(t x, \alpha_{t}\right)_{t \geq 0}$ verifies the equilibrium equations and the Kuhn-Tucker conditions, it could be not observable during a tensile test because it does not satisfy the full stability condition (12). Indeed, (30d) comes only from the first order 
stability condition (30b). To see whether the homogeneous evolution satisfies the full stability condition, let us consider a small admissible perturbation $\left(t x+h v(x), \alpha_{t}+h \beta(x)\right)$ where $h$ is a small positive real number, $v$ is the direction of perturbation of the displacement field which satisfies $v(0)=v(L)$ and $\beta \geq 0$ is the direction of perturbation of the damage field. Then, after expanding the total energy $\mathcal{P}\left(t x+h v, \alpha_{t}+h \beta\right)$ of the perturbed state up to the second order in $h$, the stability condition (12) requires that the following inequality holds

$$
0 \leq h \mathcal{P}^{\prime}\left(t x, \alpha_{t}\right)(v, \beta)+\frac{h^{2}}{2} \mathcal{P}^{\prime \prime}\left(t x, \alpha_{t}\right)(v, \beta)+o\left(h^{2}\right)
$$

where $\mathcal{P}^{\prime}\left(t x, \alpha_{t}\right)(v, \beta)$ and $\mathcal{P}^{\prime \prime}\left(t x, \alpha_{t}\right)(v, \beta)$ denote the first and second derivative of $\mathcal{P}$ at $\left(t x, \alpha_{t}\right)$ in the direction $(v, \beta)$. Specifically these derivatives read as:

$$
\begin{gathered}
\mathcal{P}^{\prime}\left(t x, \alpha_{t}\right)(v, \beta)=\int_{0}^{L}\left(\frac{1}{2} E^{\prime}\left(\alpha_{t}\right) t^{2}+w^{\prime}\left(\alpha_{t}\right)\right) \beta d x \\
\mathcal{P}^{\prime \prime}\left(t x, \alpha_{t}\right)(v, \beta)=\int_{0}^{L}\left(w_{1} \ell^{2} \beta^{\prime 2}+2 E^{\prime}\left(\alpha_{t}\right) t v^{\prime} \beta+\left(\frac{E^{\prime}\left(\alpha_{t}\right)}{2} v^{\prime 2}+w^{\prime \prime}\left(\alpha_{t}\right)\right) \beta^{2}\right) d x .
\end{gathered}
$$

Let us first remark that the states of the elastic phase are stable. Indeed, the inequality is strict in (32-a) and since the first derivative of the energy is positive in each direction $\beta \neq 0$, the inequality (37) is satisfied. Beyond the elastic phase, i.e. for $U_{t} \geq U_{e}$, the damage criterion (32-a) becomes an equality and the first derivative of the energy vanishes. It remains to study the sign of the second derivates which can also be written

$$
\begin{aligned}
& \mathcal{P}^{\prime \prime}\left(t x, \alpha_{t}\right)(v, \beta)= \\
& \int_{0}^{L} w_{1} \ell^{2} \beta^{\prime 2} d x+\int_{0}^{L} E\left(\alpha_{t}\right)\left(v^{\prime}+\frac{E^{\prime}\left(\alpha_{t}\right)}{E\left(\alpha_{t}\right)} t \beta\right)^{2} d x-\int_{0}^{L}\left(\frac{1}{2} S^{\prime \prime}\left(\alpha_{t}\right) \sigma_{t}^{2}-w^{\prime \prime}(\alpha)\right) \beta^{2} d x .
\end{aligned}
$$

The first two terms are positive. The last term of (39) is positive if and only if the behavior is with stress hardening (see (9)). Indeed, combining (35) with (9) and using $S^{\prime}\left(\alpha_{t}\right)>0$ lead to

$$
0<w^{\prime \prime}\left(\alpha_{t}\right) S^{\prime}\left(\alpha_{t}\right)-w^{\prime}\left(\alpha_{t}\right) S^{\prime \prime}\left(\alpha_{t}\right)=-S^{\prime}\left(\alpha_{t}\right)\left(\frac{1}{2} S^{\prime \prime}\left(\alpha_{t}\right) \sigma_{t}^{2}-w^{\prime \prime}\left(\alpha_{t}\right)\right) .
$$

In this case, we deduce that the state is necessarily stable. On the other hand, if the material is stress softening, the last term of (39) is negative and may induce an instability. In this case, the sign of the second derivative may be assessed through the study of the following Rayleigh ratio

$$
\mathcal{R}_{t}(v, \beta)=\frac{\int_{0}^{L} w_{1} \ell^{2} \beta^{\prime 2} d x+\int_{0}^{L} E\left(\alpha_{t}\right)\left(v^{\prime}+\frac{E^{\prime}\left(\alpha_{t}\right)}{E\left(\alpha_{t}\right)} t \beta\right)^{2} d x}{\int_{0}^{L}\left(\frac{1}{2} S^{\prime \prime}\left(\alpha_{t}\right) \sigma_{t}^{2}-w^{\prime \prime}\left(\alpha_{t}\right)\right) \beta^{2} d x} .
$$

Specifically, a sufficient (resp. necessary) condition for stability is that

$$
\rho=\min _{(v, \beta) \in \mathcal{C}_{0} \times \mathcal{D}} \mathcal{R}_{t}(v, \beta)>(\text { resp. } \geq) 1 .
$$


After some calculations which are not reproduced here (see [9] or [42]), the infimum of the Rayleigh ratio (41) is given by

$$
\rho=\frac{\min \left\{E\left(\alpha_{t}\right) S^{\prime}\left(\alpha_{t}\right)^{2} \sigma_{t}^{2} ;\left(\pi^{2} w_{1} \frac{\ell^{2}}{L^{2}} E\left(\alpha_{t}\right)^{2} S^{\prime}\left(\alpha_{t}\right)^{4} \sigma_{t}^{4}\right)^{1 / 3}\right\}}{\frac{1}{2} S^{\prime \prime}\left(\alpha_{t}\right) \sigma_{t}^{2}-w^{\prime \prime}\left(\alpha_{t}\right)} .
$$

We may finally summarize the results on the stability of homogeneous states in the following proposition.

Property 1 (Stability of homogeneous states). In the elastic phase, $U_{t}<U_{e}$, the homogeneous state of strain-damage $\left(U_{t} x / L, 0\right)$ is stable. For $U_{t} \geq U_{e}$, if the material has a stress hardening behavior (9), then the state is stable. In the case of stress softening behavior (9), the state $\left(U_{t} x / L, \alpha_{t}\right)$ with $\alpha_{t}$ given by (34) is stable if and only if the length of the bar $L$ satisfies the inequality

$$
\frac{L^{2}}{\ell^{2}} \leq \frac{\pi^{2} w_{1} E\left(\alpha_{t}\right)^{2} S^{\prime}\left(\alpha_{t}\right)^{4} \sigma_{t}^{4}}{\left(\frac{1}{2} S^{\prime \prime}\left(\alpha_{t}\right) \sigma_{t}^{2}-w^{\prime \prime}\left(\alpha_{t}\right)\right)^{3}},
$$

where $\sigma_{t}=E\left(\alpha_{t}\right) U_{t} / L$ is the stress at the equilibrium.

Remark 3. The Property 1 points out a size effect in the stability result due to the presence of the internal length. Indeed, the value $\alpha_{t}$ of the homogeneous damage given by (34) does not depend on the length of the bar. Accordingly, small or large bars give the same stress-strain diagram for homogeneous responses. However, according to (44), for large ratio L/ $\ell$, the homogeneous state will be unstable and will not be observable during an experiment. On the contrary, small bars allow homogeneous stable states.

\subsubsection{Examples}

To illustrate the previous stability analysis, we consider four examples of damage laws.

Example 1 (A model with an elastic phase). We consider the following damage law

$$
E(\alpha)=E_{0}(1-\alpha)^{2}, \quad w(\alpha)=w_{1} \alpha .
$$

This law satisfies both the strain hardening (8) and stress softening (9) conditions for any $\alpha$. Therefore the evolution problem admits a unique homogeneous strain-damage solution. Since $w^{\prime}(0)>0$, there exists an elastic phase in the evolution problem (Section 3.2.1). Hence, using (33) and (36), the displacement and the stress at the elastic limit read

$$
\sigma_{e}=\sigma_{M}=\sqrt{w_{1} E_{0}}, \quad U_{e}=\sqrt{\frac{w_{1}}{E_{0}}} L=\frac{\sigma_{M}}{E_{0}} L .
$$

Using (34) with (45) we deduce the value of the damage at each time

$$
\alpha_{t}=\max \left(0,1-\left(\frac{U_{e}}{U_{t}}\right)^{2}\right)
$$


The associated stress-strain relation reads

$$
\sigma_{t}= \begin{cases}\sigma_{M} \frac{U_{t}}{U_{e}} & \text { if } U_{t} \leq U_{e} \\ \sigma_{M}\left(\frac{U_{e}}{U_{t}}\right)^{3} & \text { otherwise. }\end{cases}
$$

We notice that the peak stress $\sigma_{M}$ and the elastic limit stress $\sigma_{e}$ given by (33) are identical for this law. Figure 2(a) reports the stress in the bar versus the normalized end displacement $U_{t} / U_{e}$, which is proportional to the homogeneous strain $t=U_{t} / L$. After the elastic phase, the stress decreases asymptotically to 0 , a distinctive feature of stress softening.

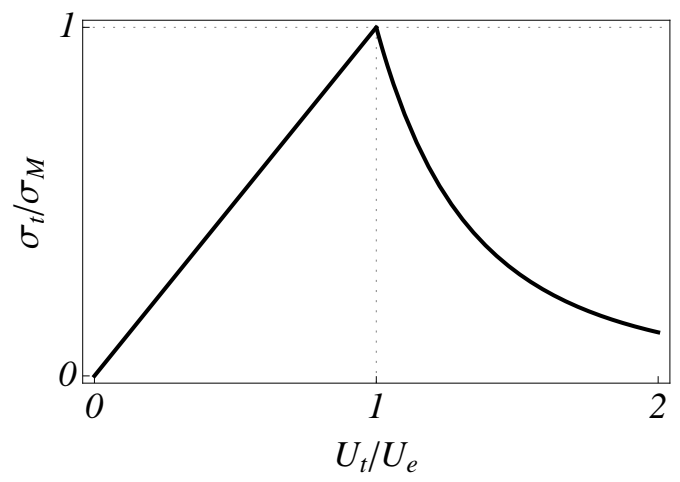

(a) Stress vs strain response

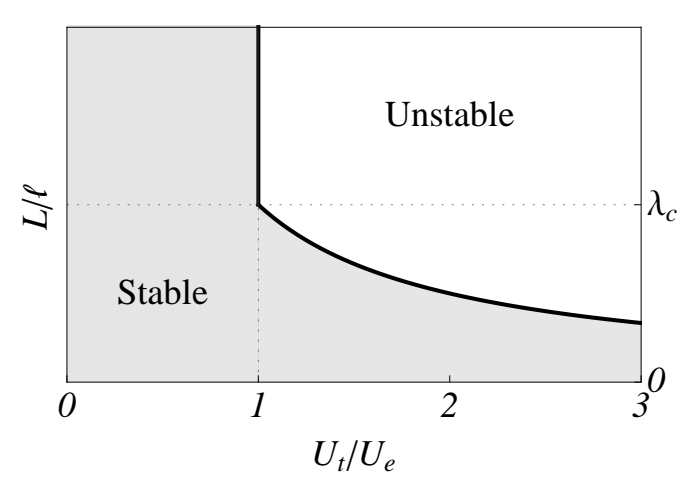

(b) Stability diagram

Figure 2: Properties of the homogeneous solutions for example 1.

Let us now discuss the stability of the homogeneous solutions. For $U_{t}<U_{e}$ the state $(t x, 0)$ is stable. For $U_{t} \geq U_{e}$, inserting (47)-(48) into (44), we deduce that the homogeneous state $\left(t x, \alpha_{t}\right)$ is stable if and only if the length of the bar L satisfies the following condition:

$$
\frac{L}{\ell} \leq \lambda_{c} \frac{U_{e}}{U_{t}}, \quad \text { with } \quad \lambda_{c}=\frac{4 \pi}{3 \sqrt{3}} .
$$

Figure 2(b) resumes these conditions on a stability diagram in the $U_{t} / U_{e}-L / \ell$ plane. Depending on the length of the bar, two different behaviors occur:

- For $L>\lambda_{c} \ell$, the homogeneous state is unstable for any $U_{t} \geq U_{e}$ and a damage localization necessarily arises at the end of the elastic phase;

- For $L<\lambda_{c} \ell$, after the elastic phase $\left(U_{t}>U_{e}\right)$, there exists a finite interval $\left[U_{e}, \lambda_{c} U_{e} \ell / L\right)$ of the prescribed displacement for which the homogeneous state is still stable. However, the homogeneous state becomes unstable for $U_{t} \geq \lambda_{c} U_{e} \ell / L$. 
Example 2 (Ambrosio-Tortorelli model : a model without an elastic phase). We consider the following damage law

$$
E(\alpha)=E_{0}(1-\alpha)^{2}, \quad w(\alpha)=w_{1} \alpha^{2},
$$

This law satisfies the strain hardening condition (8) for any $\alpha$. However the stress softening condition (9) is ensured only for $\alpha \geq 1 / 4$. Moreover since $w^{\prime}(0)=0$, this damage law does not have an elastic phase $\left(\sigma_{e}=0\right)$. Hence, using (36), the peak stress is

$$
\sigma_{M}=\frac{3 \sqrt{3}}{8 \sqrt{2}} \sqrt{w_{1} E_{0}} .
$$

From (34), we deduce that the evolution of the homogeneous damage level is given by

$$
\alpha_{t}=\frac{U_{t}^{2}}{U_{t}^{2}+3 U_{M}^{2}} \quad \text { with } \quad U_{M}=\frac{16 \sigma_{M}}{9 E_{0}} L
$$

Therefore the relation between the stress of the homogeneous solution and the prescribed displacement at $x=L$ is given by

$$
\sigma_{t}=E_{0} \frac{9 U_{M}^{4}}{\left(U_{t}^{2}+3 U_{M}^{2}\right)^{2}} \frac{U_{t}}{L} .
$$

The material becomes stress softening for $U_{t} \geq U_{M}$. The peak stress is $\sigma_{M}$ and is reached for $U_{t}=U_{M}$. Figure 3(a) shows the stress in the bar versus the homogeneous strain.

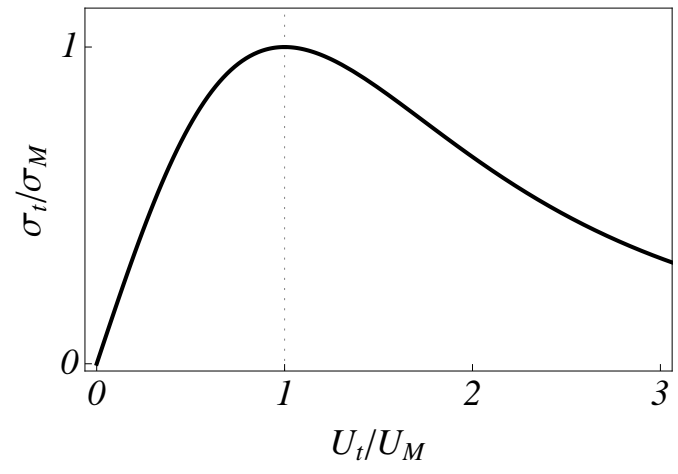

(a) Stress vs strain response

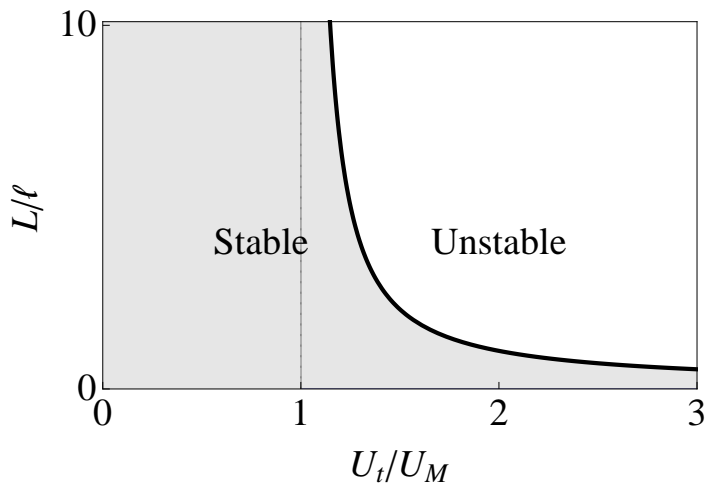

(b) Stability diagram

Figure 3: Properties of the homogeneous solutions for example 2.

In this case, the stability of the state is ruled by the second derivative of the energy. Indeed, there is not an elastic phase, and the first derivative of the potential energy is always zero. During 
the stress hardening phase $\left(U_{t}<U_{M}\right)$, the homogeneous state is stable no matter the length of the bar. Beyond $U_{M}$, the bar is in a stress softening regime and we can apply Property 1: the state is stable if the length of the bar verifies the following condition

$$
\frac{L}{\ell} \leq \frac{\pi \sqrt{3}}{4} \frac{U_{t}^{2} / U_{M}^{2}}{\left(U_{t}^{2} / U_{M}^{2}-1\right)^{3 / 2}} .
$$

The stability diagram is plotted on Figure 3(b). Beyond the stress hardening regime $\left(U_{t} \geq U_{M}\right)$, whatever the choice of the length ratio $L / \ell$, size effects rule the stability of the solution. For sufficient large displacements (the critical value depending on the bar length), the homogeneous state becomes unstable and a localization arises somewhere in the bar.

Example 3 (A family of models with the same homogeneous strain-stress response). We consider the following family of damage models indexed by the parameter $p>0$ :

$$
E(\alpha)=E_{0}(1-\alpha)^{p}, \quad w(\alpha)=w_{1}\left(1-(1-\alpha)^{p / 2}\right) .
$$

This case is a generalization of the law (45) which is recovered for $p=2$. It satisfies both the strain hardening (8) and stress softening (9) conditions for any $\alpha \in[0,1)$ and any $p>0$. Since $w^{\prime}(0)>0$, the damage evolution contains an elastic phase (Section 3.2.1) and there exists a unique homogeneous strain-damage solution. Hence, using (33) and (36), the displacement and the stress at the elastic limit read

$$
\sigma_{e}=\sigma_{M}=\sqrt{w_{1} E_{0}}, \quad U_{e}=\sqrt{\frac{w_{1}}{E_{0}}} L=\frac{\sigma_{M}}{E_{0}} L .
$$

Using (34) with (55) we deduce the value of the damage at each time

$$
\alpha_{t}=\max \left(0,1-\left(\frac{U_{e}}{U_{t}}\right)^{4 / p}\right) .
$$

The corresponding stress is

$$
\sigma_{t}= \begin{cases}\sigma_{M} \frac{U_{t}}{U_{e}} & \text { if } U_{t} \leq U_{e} \\ \sigma_{M}\left(\frac{U_{e}}{U_{t}}\right)^{3} & \text { otherwise. }\end{cases}
$$

Therefore, this family of damage laws, indexed by p, leads to the same strain-stress diagram whatever the value of $p$.

This example points out that a force-displacement diagram obtained from a tensile test is not sufficient to identify the damage model. Indeed, the force-displacement response only involves one combination of the two state functions $(E(\alpha)$ and $w(\alpha))$. At least another experimental curve is necessary. We claim that the stability analysis provides the missing information. Indeed, 


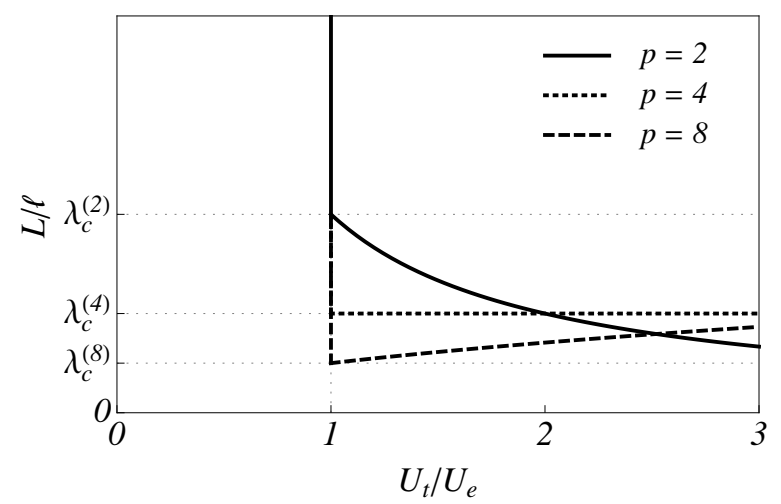

Figure 4: Stability diagram for Example 3 with $p=2,4,8$.

even if the homogeneous (elastic) states are stable for $U<U_{e}$, for $U \geq U_{e}$ the stability of an homogeneous state depends both on the length of the bar and on p. Specifically, the homogeneous state $\left(t x, \alpha_{t}\right)$ is stable when $U_{t} \geq U_{e}$ if and only if

$$
\frac{L}{\ell} \leq \lambda_{c}^{(p)}\left(\frac{U_{e}}{U_{t}}\right)^{4 / p-1}, \text { with } \quad \lambda_{c}^{(p)}=\frac{8 \pi}{3 \sqrt{3} p} .
$$

Therefore the stability diagram depends on p, cf Figure 4:

- When $p<4$, for sufficient large displacements (the critical value depending on the bar length) the homogeneous state becomes unstable.

- When $p>4$, if $L>\lambda_{c}^{p} \ell$, all homogeneous states $\left(U_{t} x / L, \alpha_{t}\right)$ with $U_{t}$ in the interval $\left[U_{e}, U_{c}^{(p)}\right)$ being

$$
U_{c}^{(p)}=\left(\frac{\lambda_{c}^{(p)} \ell}{L}\right)^{\frac{p}{4-p}} U_{e} .
$$

are unstable. However, the homogeneous states are stable again for $U_{t} \geq U_{c}^{(p)}$.

This example underlines the relevance of the stability properties in the identification the damage laws $E(\alpha)$ and $w(\alpha)$.

Example 4 (A model where ultimate fracture occurs at finite strain). With all the previous models, the ultimate fracture occur at infinite strain in a homogeneous path. We now consider a model which breaks at a finite strain (see [1]). It is defined by the following material functions parametrized by a scalar parameter $k>1$

$$
E(\alpha)=\frac{1-\alpha}{1+(k-1) \alpha} E_{0}, \quad w(\alpha)=w_{1} \alpha, \quad k>1 .
$$


For such model, a direct calculation using (33) and (60) gives

$$
\sigma_{e}=\sigma_{M}=\sqrt{\frac{2 w_{1} E_{0}}{k}}, \quad U_{e}=\sqrt{\frac{2 w_{1}}{k E_{0}}} L=\frac{\sigma_{M}}{E_{0}} L .
$$

Using (34) with (60) we deduce the value of the damage at each time

$$
\alpha_{t}=\max \left(0, \frac{1}{k-1}\left(\frac{U_{t}}{U_{e}}-1\right)\right)
$$

We deduce that for such model, the ultimate damage value is reached for a finite displacement $U_{t}=k U_{e}$. The corresponding stress is piecewise linear and reads

$$
\sigma_{t}= \begin{cases}\sigma_{M} \frac{U_{t}}{U_{e}} & \text { if } U_{t} \leq U_{e}, \\ \frac{\sigma_{M}}{k-1}\left(k-\frac{U_{t}}{U_{e}}\right) & \text { if } U_{e} \leq U_{t} \leq k U_{e} .\end{cases}
$$

Let us now focus on the stability properties of this model. For $U_{t}<U_{e}$ the state $(t x, 0)$ is stable. For $U_{t} \geq U_{e}$, inserting (62) into (44), we deduce that the homogeneous state $\left(t x, \alpha_{t}\right)$ is stable if and only if the length of the bar $L$ satisfies the following condition:

$$
\frac{L}{\ell} \leq \lambda_{c}^{(k)} g^{(k)}\left(\frac{U_{e}}{U_{t}}\right), \quad \text { with } \quad \lambda_{c}^{(k)}=\frac{\pi k}{\sqrt{2}} \quad \text { and } \quad g^{(k)}: x \mapsto \sqrt{\frac{1}{(k-1) x}\left(\frac{k}{x}-1\right)} .
$$

As in previous examples, two different behaviors occur depending on the bar length:

- For $L>\lambda_{c}^{(k)} \ell$, the homogeneous state is unstable for any $U_{t} \geq U_{e}$ and a damage localization necessarily arises at the end of the elastic phase;

- For $L<\lambda_{c}^{(k)} \ell$, after the elastic phase $\left(U_{t}>U_{e}\right)$, there exists a finite interval $\left[U_{e}, U_{e} f^{(k)}\left(\frac{\lambda_{c}^{(k)} \ell}{L}\right)\right)$ of the prescribed displacement with $f^{(k)}=\frac{1}{\left[1 / g^{(k)}\right]^{-1}}$ for which the homogeneous state is still stable. However, the homogeneous state becomes unstable for $U_{t} \geq U_{e} f^{(k)}\left(\frac{\lambda_{c}^{(k)} \ell}{L}\right)$.

\subsection{Non homogeneous evolution with damage localization}

When a homogeneous state looses its stability, a localization of the damage field arises over a finite length controlled by the internal length $\ell$ of the model. We study here the properties of solutions with damage localization by focusing on the class of stress-softening material with an elastic phase. We only indicate the main lines of the construction of the localized solutions. The interested reader should refer to [40] for the proofs and the details of the calculations. 


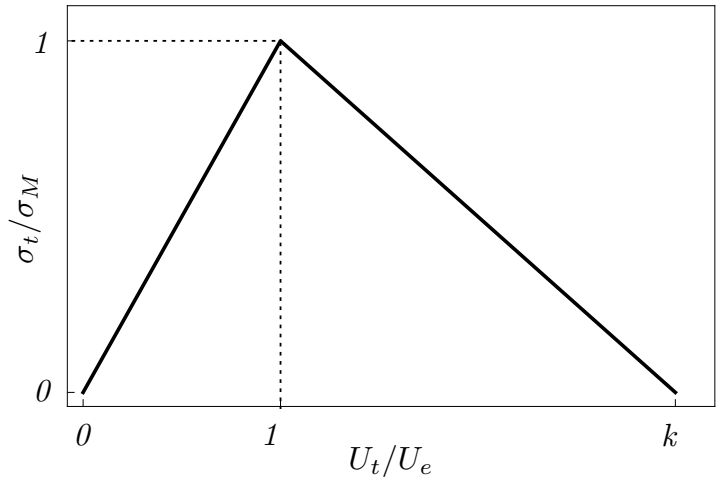

(a) Stress vs strain response

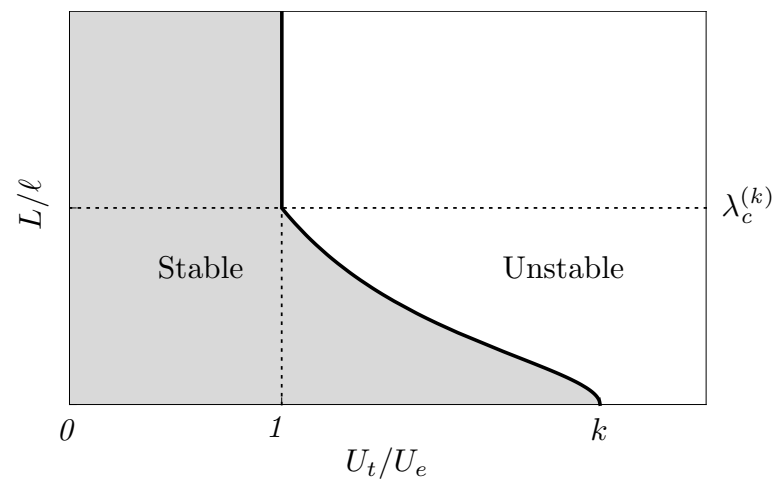

(b) Stability diagram

Figure 5: Properties of the homogeneous solutions for example 4.

\subsubsection{Optimal damage profile}

Let $\sigma \in\left(0, \sigma_{M}\right)$ be the equilibrium stress of the bar and $\mathcal{S}=\left(x_{0}-D, x_{0}+D\right)$ be a localization zone, where the thickness $D$ has to be determined and $x_{0}$ is an arbitrary point of the bar. To construct the damage profile in the localization zone, we suppose that the damage criterion (30b) is an equality only on $\mathcal{S}$ and that the damage is zero on the remaining part of the bar. Accordingly, the damage field $\alpha$ satisfies

$$
-\sigma^{2} S^{\prime}(\alpha)+2 w^{\prime}(\alpha)-2 w_{1} \ell^{2} \alpha^{\prime \prime}=0 \quad \text { on } \quad \mathcal{S}, \quad \alpha=0 \quad \text { on } \quad(0, L) / \mathcal{S} .
$$

Since $\alpha$ and $\alpha^{\prime}$ must be continuous ${ }^{2}$ at $x_{0} \pm D$, we have

$$
\alpha\left(x_{0} \pm D\right)=\alpha^{\prime}\left(x_{0} \pm D\right)=0 .
$$

Multiplying (65) by $\alpha^{\prime}$ and integrating with respect to $x$, we obtain the first integral

$$
-\sigma^{2} S(\alpha)+2 w(\alpha)-w_{1} \ell^{2} \alpha^{2}=C \text { in } \mathcal{S},
$$

where $C$ is a constant. Evaluating (67) at $x_{0} \pm D$, the conditions (66) and Hypothesis 2.2 give $C=-\sigma^{2} / E_{0}$. Hence the first integral may be written in the form

$$
\ell^{2} \alpha^{\prime}(x)^{2}=H(\sigma, \alpha(x)) \quad \text { in } \quad \mathcal{S}
$$

where

$$
H(\sigma, \beta):=\frac{2 w(\beta)}{w_{1}}-\frac{\sigma^{2}}{w_{1} E_{0}}\left(E_{0} S(\beta)-1\right), \quad \text { with } \beta \in[0,1)
$$

\footnotetext{
${ }^{2}$ The continuity of $\alpha^{\prime}$ at $x_{0} \pm D$ is obtained as a first order optimality condition on $\mathcal{P}(u, \alpha)$.
} 

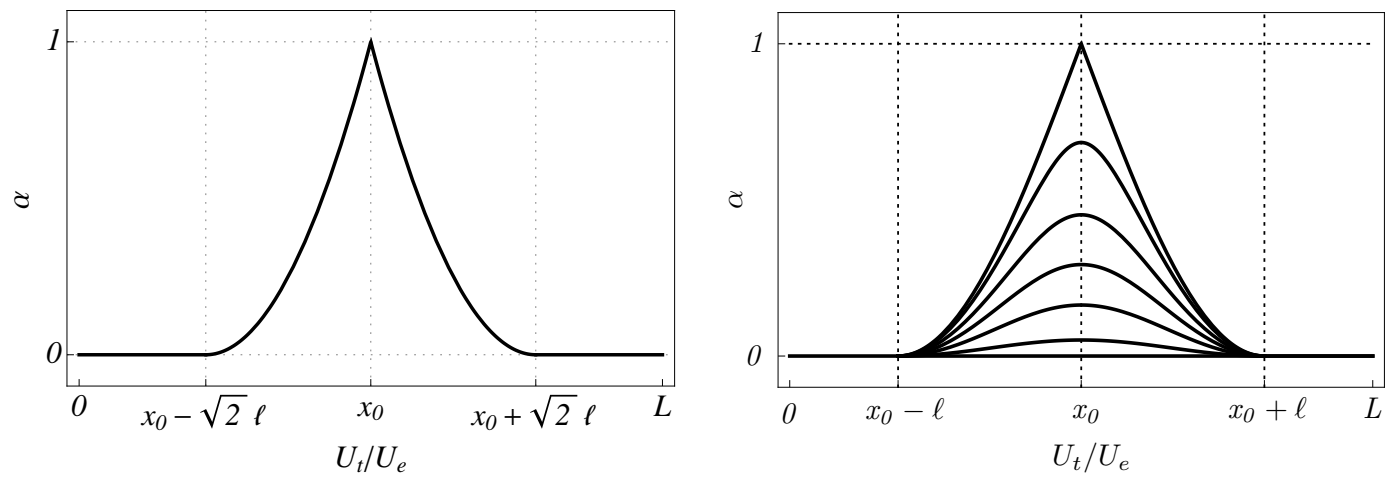

Figure 6: (Left) The damage profile $\alpha(x)$ centered at $x_{0}$ for $\sigma=0$ when considering the damage law (45), see equation (77). (Right) Evolution of the damage profile when $\sigma$ goes from $\sigma_{M}$ to 0 for the damage model considered in [1]. For such choice, the size of the damage localization is $2 \ell$ and remains constant during the localization process.

The localized damage profile is the cusp-shaped curve reported in Figure 6. We define $\bar{\alpha}(\sigma)$ as the maximal value of the damage along the bar at the given stress value $\sigma$, which is attained at $x_{0}$, the center of the localization zone. This maximal value depends only on $\sigma$. When $\sigma$ decreases from $\sigma_{M}$ to $0, \bar{\alpha}(\sigma)$ increases from 0 to 1 .

The size $D_{0}$ of the localization zone is deduced from (68) by integration. It is a function of the stress $\sigma$ :

$$
D(\sigma)=\ell \int_{0}^{\bar{\alpha}(\sigma)} \frac{d \beta}{\sqrt{H(\sigma, \beta)}} .
$$

For the assumed constitutive behavior, the integral above is well defined and always exists. The position $x_{0}$ of the center of the localized solution can be chosen arbitrarily in the interval $[D(\sigma), L-D(\sigma)]$. We finally deduce from (68) that, in the localization zone, the damage field is given by the following implicit relation between $x$ and $\alpha$ :

$$
\left|x-x_{0}\right|=\ell \int_{\alpha}^{\bar{\alpha}(\sigma)} \frac{d \beta}{\sqrt{H(\sigma, \beta)}} .
$$

It is easy to see that the damage field is symmetric with respect to the center of the localization zone, decreasing continuously from $\bar{\alpha}(\sigma)$ at the center to 0 at the boundary (see Figure 6 ).

\subsubsection{Energetic interpretation of a damage localization and link with fracture toughness}

We give in this section an energetic view of a localization process to allow for a consistent definition of fracture toughness. The total energy of a bar with a single damage localization at 
centered around $x_{0}$ with a width $2 D(\sigma)$. Rewriting the total energy of the system in terms of the stress state, we find that

$$
\mathcal{P}(u, \alpha)=\int_{0}^{L}\left(\frac{1}{2} w_{1} \ell^{2} \alpha^{\prime 2}+\frac{1}{2} S(\alpha) \sigma^{2}+w(\alpha)\right) d x .
$$

Making use of the first integral (68) and the fact that $\alpha=0$ (elastic unloading) outside the localized zone, we have

$$
\mathcal{P}(u, \alpha)=\frac{1}{2} \frac{\sigma^{2}}{E_{0}} L+\int_{x_{0}-D(\sigma)}^{x_{0}+D(\sigma)} 2 w(\alpha(x)) d x .
$$

Recalling the symmetry of the profile $\alpha$ over $\left[x_{0}-D(\sigma), x_{0}+D(\sigma)\right]$ and performing the change of variable $y=\alpha(x)$, we obtain

$$
\mathcal{P}(u, \alpha)=\frac{1}{2} \frac{\sigma^{2}}{E_{0}} L+4 \ell \int_{0}^{\bar{\alpha}(\sigma)} \frac{w(\beta) d \beta}{\sqrt{H(\sigma, \beta)}} .
$$

The link with the fracture toughness is made by passing to the limit $\sigma=0$ which corresponds to the nucleation of a macroscopic crack. In this limit, the damage localization profile is given by the following equations after taking the limit in the first integral (68) and localization width $(70)$

$$
\bar{\alpha}(0)=1, \quad \ell^{2} \alpha^{\prime}(x)^{2}=\frac{2 w(\alpha)}{w_{1}}, \quad D_{1}:=D(0)=\ell \int_{0}^{1} \sqrt{\frac{w_{1}}{2 w(\beta)}} d \beta .
$$

Now passing to the limit in the total energy (74), the first term vanishes while the second term tends to

$$
G_{c}=2 \ell \int_{0}^{1} \sqrt{2 w_{1} w(\beta)} d \beta=c_{w} \frac{\ell w_{1}}{\sqrt{2}} \quad \text { with } c_{w}=4 \int_{0}^{1} \sqrt{\frac{w}{w_{1}}} d \beta .
$$

As a result of a full localization process, $G_{c}$ is the energy dissipated in the creation of a single crack and therefore is identified as the fracture toughness of the material involved in Griffith theory of brittle fracture. Such fundamental feature allows for a use of gradient damage model to approximate brittle fracture as it will be seen in Section 4.

Remark The expression (76) for $G_{c}$ is valid for any gradient damage model presented in Section 2. Therefore the fracture energy $G_{c}$ is finite (neither zero nor infinite) provided that the internal length $\ell$ is not zero and the specific damage energy $w_{1}$ is finite (even if the strain at failure in the homogeneous response is infinite). Accordingly, $G_{c}$ is finite for all the four models presented in Section 3.2.4.

\subsubsection{Application to concrete}

To illustrate the link between fracture toughness and damage models for a specific material such as concrete, we consider a damage model for which the dissipated material function is a 
linear function of $\alpha$ i.e. $w(\alpha)=w_{1} \alpha$. Such choice applies to the models (45) of Example 1 and (60) of Example 4. In this case, the profile of a damage localization when $\sigma=0$ is given by solving the differential equation (75)

$$
\alpha(x)=\left\{\begin{array}{l}
\left(1-\frac{\left|x-x_{0}\right|}{\sqrt{2} \ell}\right)^{2} \\
0 \quad \text { elsewhere. }
\end{array} \text { if } x \in\left[x_{0}-D_{1}, x_{0}+D_{1}\right],\right.
$$

$\alpha$ being zero elsewhere (see Figure 6). The fracture toughness, the damage localization halfwidth when fracture occurs and the elastic limit stress are computed from (75)-(76) and read

$$
G_{c}=\frac{4 \sqrt{2}}{3} w_{1} \ell, \quad c_{w}=\frac{8}{3}, \quad D_{1}=\sqrt{2} \ell, \quad \sigma_{M}=\sqrt{w_{1} E_{0}} .
$$

Therefore, given a set of constitutive functions, the internal length of a gradient damage model is determined from the knowledge of the fracture toughness $G_{c}$, the elastic limit stress $\sigma_{M}$ and the Young modulus $E_{0}$. For a brittle material such as concrete, typical values for material parameters are (see e.g. [16]):

$$
E_{0}=29 \mathrm{GPa}, \quad \sigma_{M}=4.5 \mathrm{MPa}, \quad G_{c}=70 \mathrm{~N} / \mathrm{m} .
$$

Using relations (78), we find

$$
2 D_{1}=106 \mathrm{~mm}, \quad \ell=38 \mathrm{~mm}, \quad w_{1}=698 \mathrm{~N} / \mathrm{m}^{3} .
$$

Such values are consistent with the order of magnitude of the aggregates size in concrete which range from $10 \mathrm{~mm}$ to $40 \mathrm{~mm}$.

\section{Application to the numerical simulation of brittle fracture problems}

\subsection{Approximation of variational brittle fracture}

In this Section we illustrate how the damage models presented in this paper allow to capture a wide variety of phenomena in damage and fracture mechanics including diffuse damage, multiple cracks branching as well as complex crack topologies through a series of examples. To show how such gradient damage models are suitable for the simulation of brittle fracture mechanics, let us first rewrite the total energy of the system (11) for a body $\Omega$, assuming no external body and surface forces for brevity, by introducing the fracture toughness energy $G_{c}$ defined in (76):

$$
\mathcal{P}(\mathbf{u}, \alpha)=\int_{\Omega} \frac{1}{2} \mathrm{~A}(\alpha)\left(\varepsilon(\mathbf{u})-\varepsilon^{0}\right) \cdot\left(\varepsilon(\mathbf{u})-\varepsilon^{0}\right) d \Omega+\frac{G_{c}}{c_{w}} \int_{\Omega}\left(\frac{1}{\tilde{\ell}} \frac{w(\alpha)}{w_{1}}+\tilde{\ell} \nabla \alpha \cdot \nabla \alpha\right) d \Omega
$$

where we used the relation (78) between the energy dissipated in a full localization $G_{c}$ and the specific damage energy $w_{1}$ and where we set $\tilde{\ell}=\ell / \sqrt{2}$ to maintain the equivalence with the 
notation used in other works. Such energy functional can be viewed as a regularization of the Griffith model. Indeed, in fracture mechanics approaches, material failure is modeled by the nucleation and propagation of surfaces of discontinuity of the displacement field. Considering a similar solid $\Omega$ where the network of cracks $^{3}$ is denoted by $\Gamma$, the quasi-static evolution problem of fracture mechanics consists in finding the displacement field $\mathbf{u}$ and the crack set $\Gamma$ as a function of the loading. In the variational approach to brittle fracture, such evolution problem is formulated as a global minimization problem of the following Griffith energy functional, under an irreversibility condition for the crack set $\Gamma[19]$ :

$$
\mathcal{F}(\mathbf{u}, \Gamma)=\int_{\Omega \backslash \Gamma} \frac{1}{2} \mathrm{~A}_{0}\left(\varepsilon(\mathbf{u})-\varepsilon^{0}\right) \cdot\left(\varepsilon(\mathbf{u})-\varepsilon^{0}\right) d \Omega+G_{c} \mathcal{H}(\Gamma)
$$

where the material constants of the Griffith model reduce to $\mathrm{A}_{0}$ and $G_{c}$, the sound stiffness tensor and the fracture toughness of the brittle material, respectively. In (82), the total energy of the system is the sum of the elastic energy stored in the cracked body $\Omega \backslash \Gamma$ and the surface energy required to create the crack, $\mathcal{H}$ being the measure of $\Gamma$, i.e. the total crack length for $n=2$ or the total crack surface for $n=3$. A fundamental mathematical result [14] shows that the minimizers of the total energy of the gradient damage models rewritten as (81) converge when $\tilde{\ell}$ tends to 0 towards the minimizers of the Griffith functional energy (82). Such property justifies rigorously the use of the gradient damage model as a consistent approximation of Griffith model of brittle fracture and will be illustrated numerically in this section through different numerical simulations.

All the simulations presented here will use the quadratic damage model of Example 1 in Section 3.2.4 defined as

$$
\mathrm{A}(\alpha)=(1-\alpha)^{2} \mathrm{~A}_{0}, \quad w(\alpha)=w_{1} \alpha .
$$

with the sound stiffness $A_{0}$ associated to a Young's modulus $E$ and a Poisson ratio $\nu$. There are two main reasons to select this model: (i) the model has a non-vanishing elastic phase; (ii) the associated functional $\mathcal{P}(\mathbf{u}, \alpha)$ turns out to be quadratic in the damage variable, a valuable feature for its numerical minimization.

\subsection{Time-discrete evolution and solution algorithm}

The numerical solution considers a discretized version of the evolution problem. Given the displacement and the damage field $\left(\mathbf{u}_{i-1}, \alpha_{i-1}\right)$ at time step $t_{i-1}$, the solution at time step $t_{i}$ is obtained by solving the following bound-constrained minimization problem

$$
\inf \left\{\mathcal{P}_{t_{i}}(\mathbf{u}, \alpha): \quad \mathbf{u} \in \mathcal{C}_{t_{i}}, \quad \alpha \in \mathcal{D}_{t_{i}}\right\}
$$

where $\mathcal{D}_{t_{i}}=\left\{\alpha \in H^{1}(\Omega): \alpha(x) \geq \alpha_{i-1}\right.$ a.e. $\}$. The unilateral constraint $\alpha(x) \geq \alpha_{i-1}$ is the time-discrete version of the irreversibility of damage. Here we assume a uniform time step $\Delta t$.

\footnotetext{
${ }^{3}$ cracks being surfaces in dimension 3 and lines in dimension 2
} 


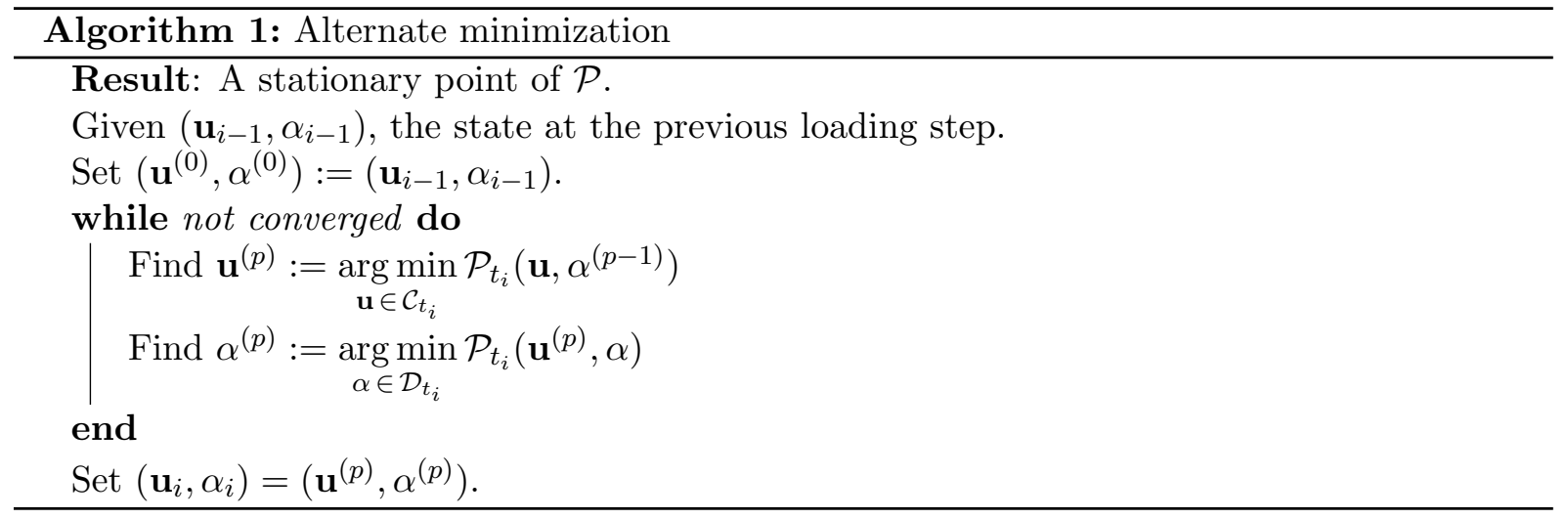

The solution of this minimization problem has been discussed in a series of papers. We use the standard alternate minimization algorithm reported in Algorithm 1. It exploits that the functional to be minimized is quadratic in each of the two variables once the other is fixed. Hence it reduces the problem to the iterative solution of quadratic minimization problems, each with a unique solution. The damage problem includes a unilateral constraint and requires the use of variational inequalities solvers, which are available in the open-source library PETSc [7]. We refer to [11] and [17] for further details and extensions of the algorithm. The problem is discretized in space with standard triangular finite elements with piecewise linear approximation for $\mathbf{u}$ and $\alpha$. Attractive anisotropic remeshing strategies specific for this minimization problem have been proposed in [5]. However, the simulations presented below are obtained on fixed meshes. The mesh size is selected to have a sufficient number of elements in the localization bands (we typically use 5-10 elements in a localization band). Open-source finite element implementations based on the FEniCS library [29] are available at [32] and [18].

\subsection{Traction of a bar}

We solve numerically the time-discrete evolution problem for the $2 \mathrm{D}$ version of the uniaxial traction test studied analytically in the previous section. We consider a bar in a plane-stress state with an aspect ratio $L=1.0, H=0.1, \ell=0.1$.

As shown in Figure 7, the numerical solution is purely elastic for $t<t_{c}=\sqrt{3 G_{c} / 8 E \ell} \simeq 1.94$ and with a single transverse localisation band (a crack) represented for $t>t_{c}$. The cracked solution has a vanishing elastic energy and a surface energy given by $G_{c} H=0.1$. The numerical results illustrate and confirm the result of the analytical study. We are in the case of long bars where the homogeneously damaged solution is unstable (see Figure 2(b)). Further numerical examples for short bars with the discussion of the associated size effects are reported in [37]. The test may be easily extended to a 3D geometry. The critical load $t_{c}$ is the same in $1 \mathrm{D}$ under a uniaxial stress condition, in 2D plane stress, or in 3D. Note the energy balance is not verified 


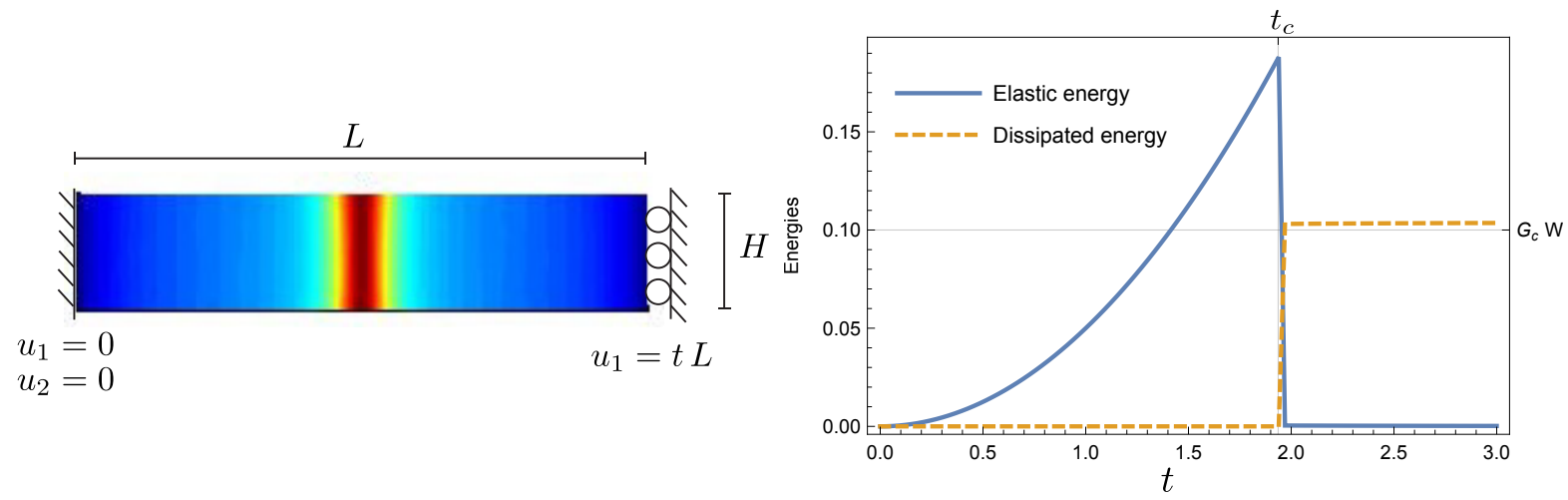

Figure 7: Uniaxial traction of bar. Left: boundary conditions and damage field for $t>t_{c}$. Right: evolution of the energy at the solution given by the alternate minimization algorithm as the applied end-displacement $t$ is increased. Reproduced from [17].

across $t=t_{c}$. Indeed, the time-discrete evolution based on the unilateral minimization of the energy, does not enforce energy conservation.

\subsection{Crack propagation}

The study of the propagation of a straight crack illustrates the equivalence with the Griffith propagation criterion for a pre-existing crack. We consider the surfing experiment proposed in [24]. A rectangular slab $\Omega=[0, L] \times[-H / 2, H / 2]$ with a preexisting crack of length $L_{c}$ is loaded by applying the following Dirichlet boundary condition on the whole external boundary

$$
\mathbf{U}\left(x_{1}, x_{2}, t\right)=\overline{\mathbf{U}}\left(x_{1}-L_{c}-v t, x_{2}\right) \quad \text { on } \partial \Omega
$$

where $\overline{\mathbf{U}}$ is the asymptotic Mode-I crack displacement of linear elastic fracture mechanics. ${ }^{4}$ The intensity of the loading is controlled by the stress intensity factor $K_{I}$. From the theory we expect that the crack propagates at the constant speed $v$ along the line $x_{2}=0$ for $K_{I}=K_{I}^{c}=\sqrt{G_{c} E}$. In the numerical experiments we set $K_{I} / K_{I}^{c}=1.0, v=1, L=2, H=1$ and $L_{c}=0.05$. Figure 8 reports the results of the corresponding numerical simulations. This test is particularly useful to verify that the dissipated energy does not depend on $\ell$ and is equal to the product of the crack length and the fracture toughness $G_{c}$. Obviously, in order for this condition to

${ }^{4}$ Denoting by $(r, \theta)$ the polar coordinates and $\left(e_{1}, e_{2}\right)$ the Cartesian unit vectors,

$$
\overline{\mathbf{U}}=\frac{K_{I}}{2 \mu} \sqrt{\frac{r}{2 \pi}}\left(\frac{3-\nu}{1+\nu}-\cos \theta\right)\left(\cos (\theta / 2) e_{1}+\sin (\theta / 2) e_{2}\right)
$$

where $\mu$ is the shear modulus, and $L_{c}$ is the length of the preexisting crack. 
hold, the discretisation should be changed with the internal length, as $\ell$ controls the width of the localization band. We typically set the mesh size to $h=\ell / 5$. For convenience we use a non-uniform mesh respecting this condition only in the band where we expect the crack to propagate, as shown in Figure 8.
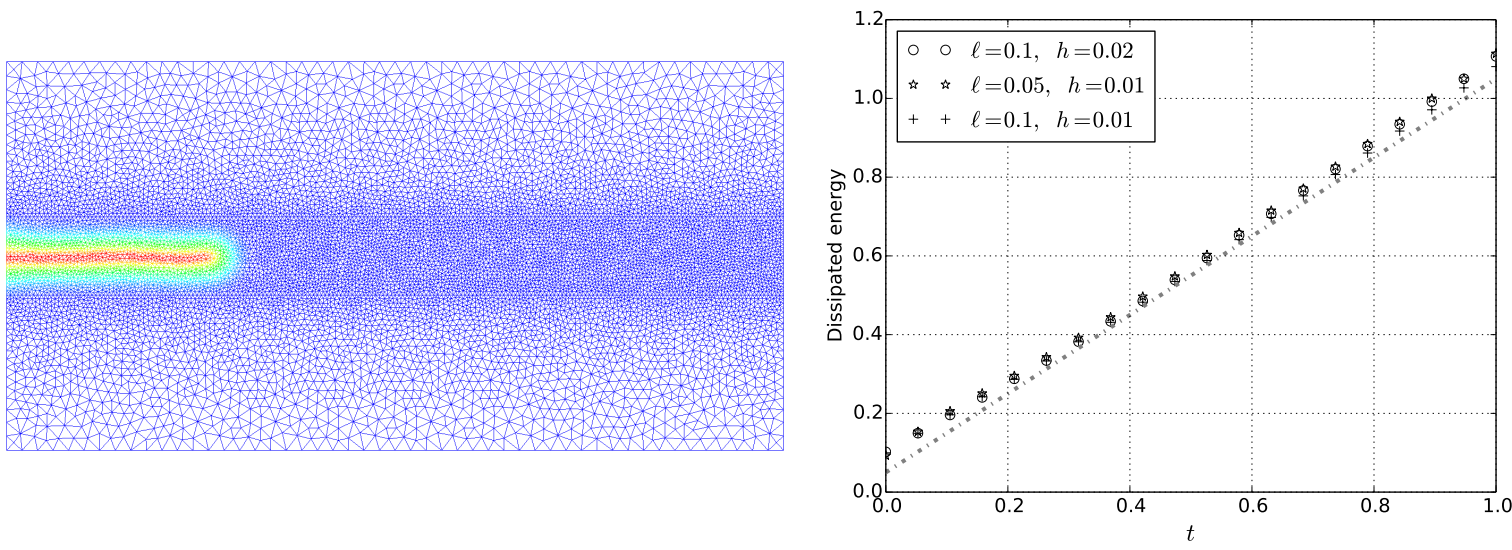

Figure 8: Smooth crack propagation test on a rectangular slab of dimensions $2 \times 1$ with the surfing loading (86) applied on the boundary. Left: snapshot of the damage field and mesh for $\ell=0.05$ and $h=0.01$. Right: Dissipation energy versus time for $v=1$ and $K_{I}=1$ comparing the results obtained through the damage model when varying the internal length $\ell$ and the mesh size $h$; the continuous line is the expected surface energy according to the Griffith model, corresponding to a constant crack speed $v=1$. Reproduced from [17].

\subsection{Thermal shock}

The shrinkage of materials, induced by cooling or drying, may lead to arrays of regularly spaced cracks. Similar phenomena appearing at very different length-scales have always intrigued researchers and common people: drying of concrete, the exposure of glass or ceramics to a thermal shock, the drying of soils, or the cooling of lava fronts with the formation of columnar joints. The understanding and the predictive simulation of the morphogenesis and propagation of similar complex crack patterns is a major issue for classical fracture mechanics, which usually studies the propagation of a single preexisting crack. Yet similar problems may be naturally tackled, theoretically and numerically, using the gradient damage models presented in this paper.

We resume here the results reported in $[13,47]$ on the thermal shock of a brittle slab. For this problem a number of experimental work are available in the literature $[6,45,21]$. The specimen is a thin slab, free at the boundary, composed of a homogeneous material without prestress in its initial configuration. In experiments, several slabs are stacked together, uniformly heated at temperature $T_{0}$ and then quenched in a cold bath inducing a temperature drop $\Delta T$ on the lateral surfaces. The resulting inhomogeneous temperature field induces an inhomogeneous stress field 
inside the slab, causing the emergence of a complex crack pattern, with an almost periodic array of cracks nucleating at the boundary and propagating inside the slab with a period doubling phenomenon.

Figure 9 consider a rectangular portion of the slab including the surface of the thermal shock. The material shrinkage induced by the thermal effects is modelled through time-dependent inelastic deformations in the form

$$
\varepsilon_{t}^{0}=\beta T_{t} I, \quad T_{t}=-\Delta T \operatorname{Erfc}\left(\frac{x_{2}}{2 \sqrt{k_{c} t}}\right),
$$

where $\beta$ is the thermal expansion coefficient, $T_{t}$ is the temperature field in the slab, and Erfc denotes the complementary error function. As a first approximation, $T_{t}$ is taken as the analytical solution of a thermal diffusion problem with a Dirichlet boundary condition on the temperature for a semi-infinite homogeneous slab of thermal diffusivity $k_{c}$, neglecting the influence of the cracks on the thermal diffusivity.

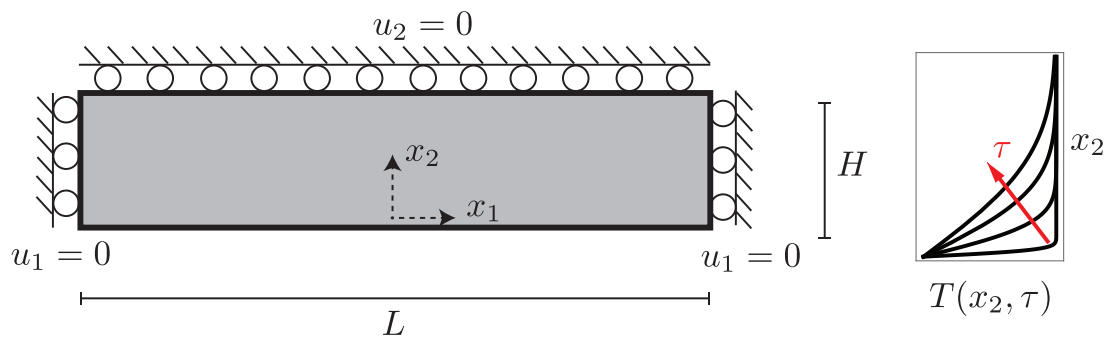

Figure 9: Geometry and boundary conditions for the thermal shock problem (left), where $u_{1}$ and $u_{2}$ denotes the two components of the displacement field. The loading is given by the thermal stress induced by the temperature field $T\left(x_{2}, \tau\right)$ of (87), whose dependence in $x_{2}$ is sketched on the right for different times $\tau$.

The dimensional analysis of the energy functional highlights three characteristic lengths: the size of the domain $L$, the internal length $\ell$, and the Griffith length $\ell_{0}=G_{c} /\left(E \beta^{2} \Delta T^{2}\right)$. Using the material's internal length as the reference unit, the problem can be reformulated in terms of two dimensionless parameters, the size of the structure $L / \ell$ (a geometric parameter) and the intensity of the thermal shock $\ell / \ell_{0}$ (a loading parameter). To put into evidence the role of the temperature drop $\Delta T$, the latter loading parameter can be replaced by

$$
\frac{\Delta T}{\Delta T_{c}}=\sqrt{\frac{8 \ell}{3 \ell_{0}}}, \quad \text { where } \quad \Delta T_{c}=\frac{1}{\beta} \sqrt{\frac{3 G_{c}}{8 E \ell}}
$$

As shown in [47], for $\Delta T<\Delta T_{c}$ the solution is purely elastic with no damage ( $\alpha=0$ everywhere). For $\Delta T>\Delta T_{c}$ the solution evolves qualitatively as in Figure 10, with (i) the immediate creation of an $x$-homogeneous damage band parallel to the exposed surface, (ii) the bifurcation of this solution toward an $x$-periodic one, which (iii) further develops in a periodic array of crack 
bands orthogonal to the exposed surface. These bands further propagate with a period doubling phenomenon (iv). A detailed analytical study of the bifurcation phenomenon observed in the phase (ii) is reported in [47]. The three columns in Figure 10 show the phases (ii)-(iv) of the evolution for $\Delta T / \Delta T_{c}$ equal to 2,4 and 8 . The wavelength of the oscillations and the spacing of the cracks increase with $\Delta T$. In particular [13] shows that for $\Delta T \gg \Delta T_{c}$ the initial crack spacing is proportional to $\sqrt{\ell_{0} \ell}$. Figure 11 reports the evolution of the dissipated energy versus time for the three cases of Figure 10. We note in particular that, while the evolution is smooth for intense thermal shocks (see the curve $\Delta T=8 \Delta T_{c}$ ), for mild shocks there are jumps in the energy dissipation and hence in the crack length (see the curve $\Delta T=2 \Delta T_{c}$ ). These jumps correspond to snap-backs in the evolution problem, where the minimization algorithm is obliged to search for a new solution, potentially far from the one at the previous time step.
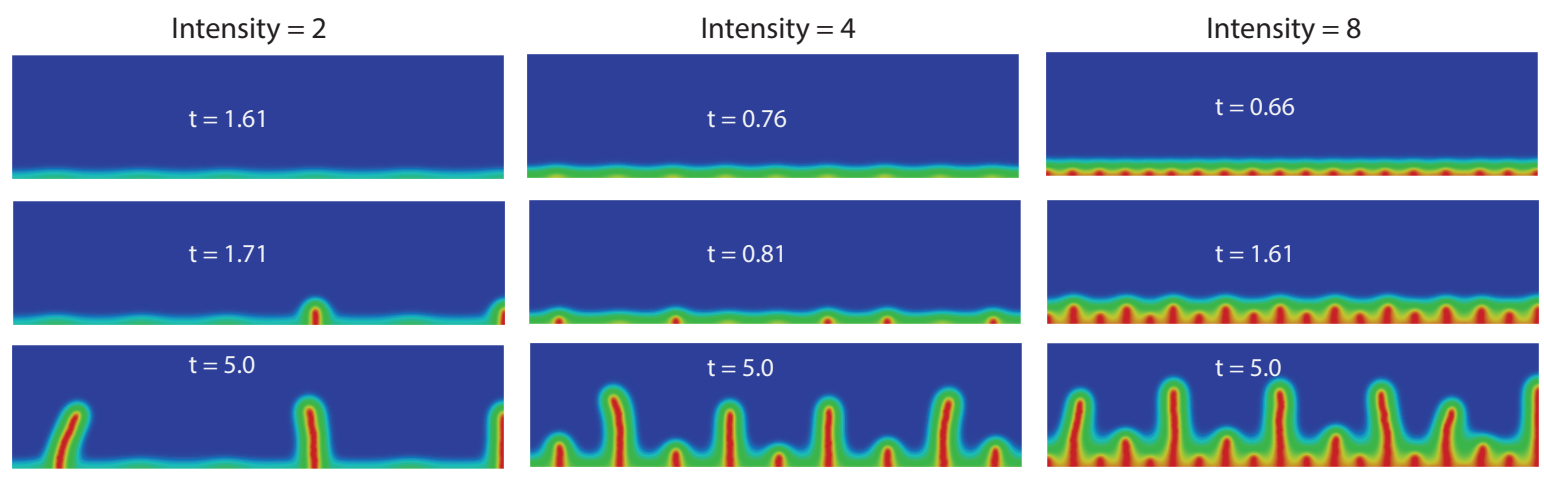

Figure 10: Evolution of the damage variable $\alpha$ during the evolution (blue: $\alpha=0$; red: $\alpha=1$ ) showing the initial solution independent of the $x_{1}$ variable, the emergence of a periodic crack pattern and its selective propagation with period doubling. Each column corresponds to the result obtained for a specific intensity, increasing from left (2) to right (8). Here $\ell=1$ and the slab dimensions are $40 \times 10$ with a mesh size $h=0.2$. Reproduced from [17].

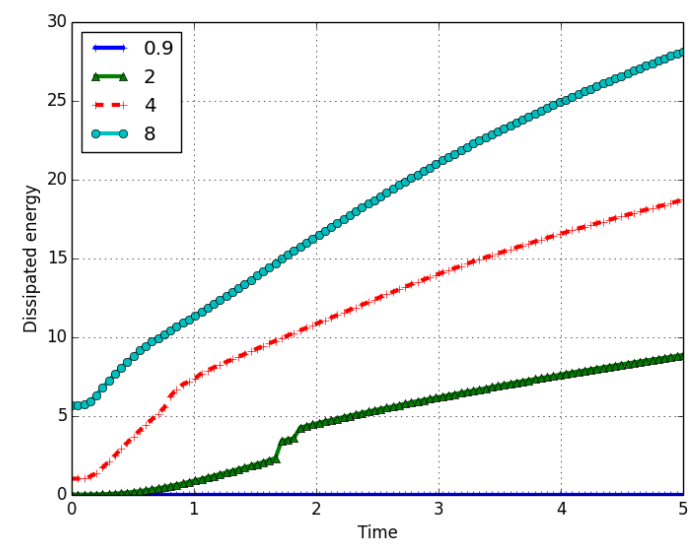

Figure 11: Evolution of the dissipated energy with time during the thermal shock for different values $\Delta T / \Delta T_{c}$ of the intensity of the shock. The time variable is put in a non-dimensional rescaled form proportional to $\sqrt{t}$. 
The three dimensional version of the thermal shock problem leads to complex crack patterns in the form of hexagonal cells of increasing diameters, which are recovered by adopted gradient damage modelling (see Figure 12). We refer the reader to [13] for further details on this problem and on the related numerical work.

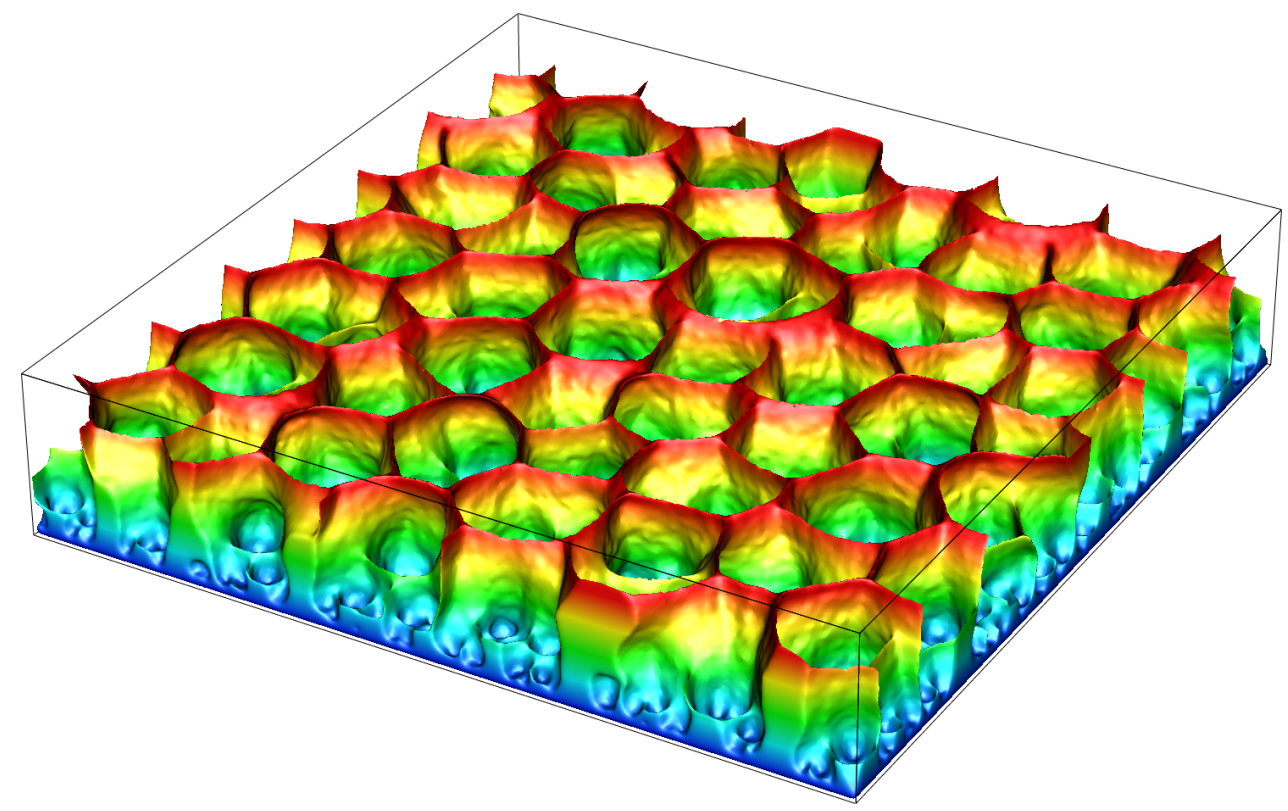

Figure 12: Complex fracture pattern for the thermal shock of brittle slab. The problem is the 3D analogue of the thermal shock problem of Figure 9. The numerical results are for $\ell_{0}=0.05 \ell$ in a domain of size $150 \ell \times 150 \ell \times 20 \ell$. Only zones with damage close to 1 are represented (cracks). To help the visualization, the colors mark the distance from the bottom surface where the thermal shock is applied. The simulation is performed using $4.4 \times 10^{7}$ linear finite elements in space and 100 time steps, using 1536 cores of the NSF-XSEDE cluster Stampede at Texas Advanced Computing Center (wall-time: 10 hours). Reproduced from [13].

\section{Concluding remarks}

We have shown in this paper that gradient damage models are good candidates to approximate the fracture of materials. Inserting them in a variational setting allows to formulate the non local damage evolution problem in a very elegant and concise manner. Moreover, the concept of stability of states rises immediately from this variational approach. Thanks to the presence a characteristic material length, we can account both for size effects and for the stability of the homogeneous states for sufficiently small bodies. The determination of the size beyond which an homogeneous state is no more stable is one of the main step in the analysis of the properties of a given model. That gives an additional information to the stress-strain curve which is fundamental to identify the state functions entering in the model. All these concepts have been developed in a full three-dimensional setting and, from a numerical viewpoint, computations of 
very complex geometry are already available.

Conflicts of interest : The authors declare that they have no conflict of interest.

\section{References}

[1] R. Alessi, J.-J. Marigo, and S. Vidoli. Nucleation of cohesive cracks in gradient damage models coupled with plasticity. Archives for Rational Mechanics and Analysis, 214:575-615, 2014 .

[2] R. Alessi, J.-J. Marigo, and S. Vidoli. Gradient damage models coupled with plasticity: variational formulation and main properties. Mechanics of Materials, 80:351-367, 2015.

[3] L. Ambrosio, N. Fusco, and D. Pallara. Functions of bounded variation and free discontinuity problems. Oxford Mathematical Monographs. Oxford Science Publications, 2000.

[4] H. Amor, J.-J. Marigo, and C. Maurini. Regularized formulation of the variational brittle fracture with unilateral contact: Numerical experiments. Journal of the Mechanics and Physics of Solids, 57(8):1209 - 1229, 2009.

[5] M. Artina, M. Fornasier, S. Micheletti, and S. Perotto. Anisotropic mesh adaptation for crack detection in brittle materials. SIAM Journal on Scientific Computing, 37(4):B633B659, 2015.

[6] H. A. Bahr, G. Fischer, and H. J. Weiss. Thermal-shock crack patterns explained by single and multiple crack propagation. J. Mater. Sci., 21:2716-2720, 1986.

[7] S. Balay, S. Abhyankar, M. F. Adams, J. Brown, P. Brune, K. Buschelman, V. Eijkhout, W. D. Gropp, D. Kaushik, M. G. Knepley, L. C. McInnes, K. Rupp, B. F. Smith, and H. Zhang. PETSc users manual. Technical Report ANL-95/11 - Revision 3.5, Argonne National Laboratory, 2014.

[8] A. Benallal, R. Billardon, and G. Geymonat. Bifurcation and localization in rate independent materials. In Q. Nguyen, editor, C.I.S.M Lecture Notes on Bifurcation and Stability of Dissipative Systems, volume 327 of International Centre for Mechanical Sciences, pages 1-44. Springer-Verlag, 1993.

[9] A. Benallal and J.-J. Marigo. Bifurcation and stability issues in gradient theories with softening. Modelling and Simulation in Material Science and Engineering, 15:S283-S295, 2007.

[10] M. J. Borden, C. V. Verhoosel, M. A. Scott, T. J. Hughes, and C. M. Landis. A phasefield description of dynamic brittle fracture. Computer Methods in Applied Mechanics and Engineering, 217-220(0):77 - 95, 2012. 
[11] B. Bourdin. Numerical implementation of the variational formulation of quasi-static brittle fracture. Interfaces and Free Boundaries, 9:411-430, 2007.

[12] B. Bourdin, G. Francfort, and J.-J. Marigo. Numerical experiments in revisited brittle fracture. Journal of the Mechanics and Physics of Solids, 48:797-826, 2000.

[13] B. Bourdin, J.-J. Marigo, C. Maurini, and P. Sicsic. Morphogenesis and propagation of complex cracks induced by thermal shocks. Phys. Rev. Lett., 112(1):014301, 2014.

[14] A. Braides. Approximation of Free-Discontinuity Problems. Lecture notes in Mathematics, vol. 1694. Springer, 1998.

[15] C. Comi. Computational modelling of gradient-enhanced damage in quasi-brittle materials. Mechanics of Cohesive-Frictional Materials, 4(1):17-36, 1999.

[16] C. Comi and U. Perego. Fracture energy based bi-dissipative damage model for concrete. International Journal of Solids and Structures, 38(36-37):6427-6454, 2001.

[17] P. Farrell and C. Maurini. Linear and nonlinear solvers for variational phase-field models of brittle fracture. International Journal of Numerical Methods in Engineering, (submitted), 2016.

[18] P. Farrell and C. Maurini. Solvers for variational damage and fracture. https:// bitbucket.org/pefarrell/varfrac-solvers, 2016.

[19] G. Francfort and J.-J. Marigo. Revisiting brittle fracture as an energy minimization problem. Journal of the Mechanics and Physics of Solids, 46(8):1319-1342, 1998.

[20] F. Freddi and G. Royer-Carfagni. Regularized variational theories of fracture : a unified approach. Journal of the Mechanics and Physics of Solids, 58:1154-1174, 2010.

[21] J. Geyer and S.Nemat-Nasser. Experimental investigations of thermally induced interacting cracks in brittle solids. Int. J. Solids Struct., 18(4):137-356, 1982.

[22] A. Giacomini. Ambrosio-Tortorelli approximation of quasi-static evolution of brittle fractures. Calculus of Variations and Partial Differential Equations, 22:129-172, 2005.

[23] V. Hakim and A. Karma. Laws of crack motion and phase-field models of fracture. Journal of the Mechanics and Physics of Solids, 57:342-368, 2009.

[24] M. Hossain, C.-J. Hsueh, B. Bourdin, and K. Bhattacharya. Effective toughness of heterogeneous media. Journal of the Mechanics and Physics of Solids, 71(0):15-32, 2014.

[25] A. Karma, D. A. Kessler, and H. Levine. Phase-field model of mode iii dynamic fracture. Phys. Rev. Lett., 87:045501, Jul 2001. 
[26] C. Kuhn and R. Müller. A continuum phase field model for fracture. Engineering Fracture Mechanics, 77(18):3625 - 3634, 2010. Computational Mechanics in Fracture and Damage: A Special Issue in Honor of Prof. Gross.

[27] G. Lancioni and G. Royer-Carfagni. The variational approach to fracture mechanics. A practical application to the French Panthéon in Paris. Journal of Elasticity, 95:1-30, 2009.

[28] B. Li, C. Peco, D. Millán, I. Arias, and M. Arroyo. Phase-field modeling and simulation of fracture in brittle materials with strongly anisotropic surface energy. International Journal for Numerical Methods in Engineering, 102(3-4):711-727, 2014.

[29] A. Logg, K.-A. Mardal, G. N. Wells, et al. Automated Solution of Differential Equations by the Finite Element Method. Springer, 2012.

[30] E. Lorentz and S. Andrieux. Analysis of non-local models through energetic formulations. International Journal of Solids and Structures, 40:2905-2936, 2003.

[31] J.-J. Marigo. Constitutive relations in Plasticity, Damage and Fracture Mechanics based on a Work Property. Nuclear Engineering and Design, 114:249-272, 1989.

[32] C. Maurini. Fenics codes for variational damage and fracture. https://bitbucket.org/ cmaurini/varfrac_for_cism, 2013.

[33] C. Miehe, M. Hofacker, and F. Welschinger. A phase field model for rate-independent crack propagation: Robust algorithmic implementation based on operator splits. Computer Methods in Applied Mechanics and Engineering, 199(45-48):2765 - 2778, 2010.

[34] D. Mumford and J. Shah. Optimal approximations by piecewise smooth functions and associated variational problems. Communications on Pure and Applied Mathematics, 42:577685, 1989.

[35] R. Peerlings, R. de Borst, W. Brekelmans, J. de Vree, and I. Spee. Some observations on localisation in non-local and gradient damage models. European Journal of Mechanics A/Solids, 15:937-953, 1996.

[36] R. Peerlings, R. de Borst, W. Brekelmans, and M. Geers. Gradient-enhanced damage modelling of concrete fracture. Mechanics of Cohesive-frictional Materialsf, 3:323-342, 1998.

[37] K. Pham, H. Amor, J.-J. Marigo, and C. Maurini. Gradient damage models and their use to approximate brittle fracture. International Journal of Damage Mechanics, 20(4):618-652, 2011.

[38] K. Pham and J.-J. Marigo. Approche variationnelle de l'endommagement: I. les concepts fondamentaux. Comptes Rendus Mécanique, 338(4):191-198, 2010. 
[39] K. Pham and J.-J. Marigo. Approche variationnelle de l'endommagement: II. Les modèles à gradient. Comptes Rendus Mécanique, 338(4):199-206, 2010.

[40] K. Pham and J.-J. Marigo. From the onset of damage until the rupture: construction of the responses with damage localization for a general class of gradient damage models. Continuum Mech. Thermodyn., 25(2-4):147-171, 2013.

[41] K. Pham and J.-J. Marigo. Stability of homogeneous states with gradient damage models: size effects and shape effects in the three-dimensional setting. J. Elasticity, 110(1):63-93, 2013. Isiweb.

[42] K. Pham, J.-J. Marigo, and C. Maurini. The issues of the uniqueness and the stability of the homogeneous response in uniaxial tests with gradient damage models. J. Mech. Phys. Solids, 59(6):1163-1190, 2011. Isiweb.

[43] A. Pons and A. Karma. Helical crack-front instability in mixed mode fracture. Nature, 464:85-89, 2010.

[44] A. Schlüter, A. Willenbücher, C. Kuhn, and R. Müller. Phase field approximation of dynamic brittle fracture. Computational Mechanics, pages 1-21, 2014.

[45] Y. Shao, X. Xu, S. Meng, G. Bai, C. Jiang, and F. Song. Crack patterns in ceramic plates after quenching. Journal of the American Ceramic Society, 93(10):3006-3008, 2010.

[46] P. Sicsic and J.-J. Marigo. From gradient damage laws to Griffith's theory of crack propagation. J. Elasticity, 113(1):55-74, 2013.

[47] P. Sicsic, J.-J. Marigo, and C. Maurini. Initiation of a periodic array of cracks in the thermal shock problem: a gradient damage modeling. J. Mech. Phys. Solids, 63(256-284), 2014. 